\title{
Linear and nonlinear regimes of an inertial wave attractor
}

\author{
Maxime Brunet, ${ }^{1}$ Thierry Dauxois, ${ }^{2}$ and Pierre-Philippe Cortet ${ }^{1, *}$ \\ ${ }^{1}$ Laboratoire FAST, CNRS, Université Paris-Sud, Université Paris-Saclay, 91405 Orsay, France \\ ${ }^{2}$ Université de Lyon, ENS de Lyon, Université Claude Bernard, \\ CNRS, Laboratoire de Physique, 69342 Lyon, France
}

(Received 31 October 2018; published 5 March 2019)

\begin{abstract}
We present an experimental analysis of the linear and nonlinear regimes of an attractor of inertial waves in a trapezoidal cavity under rotation. Varying the rotation rate and the forcing amplitude and wavelength, we identify the scaling laws followed by the attractor amplitude and wavelength in both regimes. In particular, we show that the nonlinear scaling laws can be well described by replacing the fluid viscosity in the linear model by a turbulent viscosity, a result that could help in extrapolating attractor theory to geophysically and astrophysically relevant situations. We further study the triadic resonance instability of the attractor which is at the origin of the turbulent viscosity. We show that the typical frequencies of the subharmonic waves produced by the instability behave very differently from previously reported numerical results and from the prediction of the theory of triadic resonance. This behavior might be related to the deviation from the horizontal invariance of the attractor in our experiment in relation to the presence of vertical walls of the cavity, an effect that should be at play in all practical situations.
\end{abstract}

DOI: 10.1103/PhysRevFluids.4.034801

\section{INTRODUCTION}

Fluids submitted to a global rotation enable the propagation of a specific class of waves, called inertial waves [1] as a result of the restoring action of the Coriolis force. In an inertial wave, energy propagates in a direction tilted by an angle $\theta$ with respect to the horizontal, which is defined by the dispersion relation

$$
\sigma=2 \Omega \cos \theta,
$$

where $\sigma$ is the wave angular frequency and $\Omega$ the fluid rotation rate around the vertical axis. Inertial waves are cousins of internal waves of gravity propagating in linearly stratified fluids [2]: They have similar dispersion relations linking the ratio between the wave frequency and the rotation rate or the buoyancy frequency to a specific direction along which their energy propagates. These dispersion relations lead to orthogonal group and phase velocities and also let the length scales of the wave be unprescribed by the frequency. These length scales (wavelength and beam width) are consequently set by boundary conditions, viscous dissipation, and eventually nonlinearities. This leads to a variety of wave structures such as self-similar wave beams [3-6], plane waves [7,8], and resonant cavity modes [9-13]. These waves are relevant in geophysics and astrophysics in which they often merge into inertia-gravity waves with a single dispersion relation coupling rotation and buoyancy $[2,14]$. Considering pure inertial and pure gravity waves, a major difference however exists: Gravity waves involve rectilinear fluid oscillations in the plane tilted by the angle $\theta$, whereas in inertial waves fluid particles describe anticyclonic circular translations in this plane.

\footnotetext{
*ppcortet@fast.u-psud.fr
} 
In closed domains, modes of standing waves can be found for specific frequencies when the walls are normal and parallel to the rotation axis $[1,10]$ but also in some geophysically and astrophysically relevant geometries such as spheres and spheroids [15]. In geophysics and astrophysics, several types of global forcing may be at the origin of such modes. For example, modes can be excited in spheres and spherical shells by a longitudinal libration [9,16-19] consisting in a time modulation of the rotation rate. Precession [13,20-22] and tidal deformation of the planet crust [23,24] are other examples.

However, closed domains generally include sloping walls in which case the wave focusing and defocusing induced by the peculiar reflection laws of inertial or internal gravity waves [25] prevent the existence of cavity eigenmodes [26]. It is a consequence of the dispersion relation: The wave keeps constant its propagation angle (in absolute value) with respect to the horizontal when reflecting on a wall. This implies, for reflection on tilted walls, that Snell-Descartes laws are not verified and that the wave length scales are enhanced or reduced depending on the fact that the wave is descending or climbing the slope [25]. This peculiar physics can lead to the emergence of limit cycles, called wave attractors [26-29], on which the waves concentrate when excited in closed domains with tilted walls, including the astrophysically relevant case of spherical shells [28]. From a theoretical point of view, tracing rays respecting the reflection laws directly reveals the existence (or the absence) of an attractor for a given wave frequency, via the convergence (or not) of all rays towards a unique limit cycle. Inviscid attractors actually exist over specific frequency ranges depending on the cavity geometry [26-29].

Clear experimental observations of attractors were done in trapezoidal cavities by Maas and co-workers both for internal gravity waves [27] and for inertial waves [29-31] (and also later in another geometry by Klein et al. [32] for inertial waves). In these experiments, the wave forcing was realized through a global motion of the water tank: a longitudinal libration in the case of rotation and an oscillating vertical translation in the case of stratification. In reality, the energy injected at the forcing frequency $\sigma_{0}$ does not focus on an infinitely thin parallelogram because of viscous dissipation. The scales (width and wavelength) of the attractor then follow from the competition of the energy focusing during reflections on tilted walls and the energy dissipation during wave propagation: Focusing reduces scales whereas dissipation preferentially damps small scales. It has been proposed that the attractor wave beam once unwrapped can be described as a self-similar wave beam emitted by a virtual point source located upstream of the focusing reflection [28,33-35]. This model, in which the scale reduction at the focusing reflection is exactly compensated by the viscous spreading of the beam emitted by the point source [5,6], has been tested with increasing success in numerical simulations $[35,36]$.

Since in geophysics and astrophysics extremely large Reynolds numbers are involved, a particularly interesting, but not thoroughly discussed, facet of this problem is how the attractor is affected by nonlinearities when increasing the forcing amplitude. This question has started to be explored experimentally by Scolan et al. [37] and Brouzet et al. [38] in stratified fluids and by Jouve and Ogilvie [36] in direct numerical simulations of a rotating tilted square: They have revealed the emergence of an instability of the attractor feeding two subharmonic waves in triadic resonance with the attractor. This nonlinear process has further been shown to affect the attractor by damping its amplitude and increasing its scales. These observations have been proposed to result from the additional effective dissipation that the instability induces for the attractor [36,38]. However, no scaling laws have been identified to describe the nonlinear evolution of the attractor. Even in the linear regime, it is not clear how the attractor amplitude scales with the forcing Reynolds and Rossby numbers.

In this article we propose such scaling laws and compare them successfully with experimental data. We explore the linear and nonlinear regimes of an inertial wave attractor in a trapezoidal cavity under rotation using a corotating particle image velocimetry system. The flow is generated by a deformable upper cover of the cavity which has a sinusoidal shape with a phase propagating horizontally. The dependences of the scale and amplitude of the attractor are studied as a function of the forcing amplitude for several rotation rates and forcing wavelengths. This large data set 


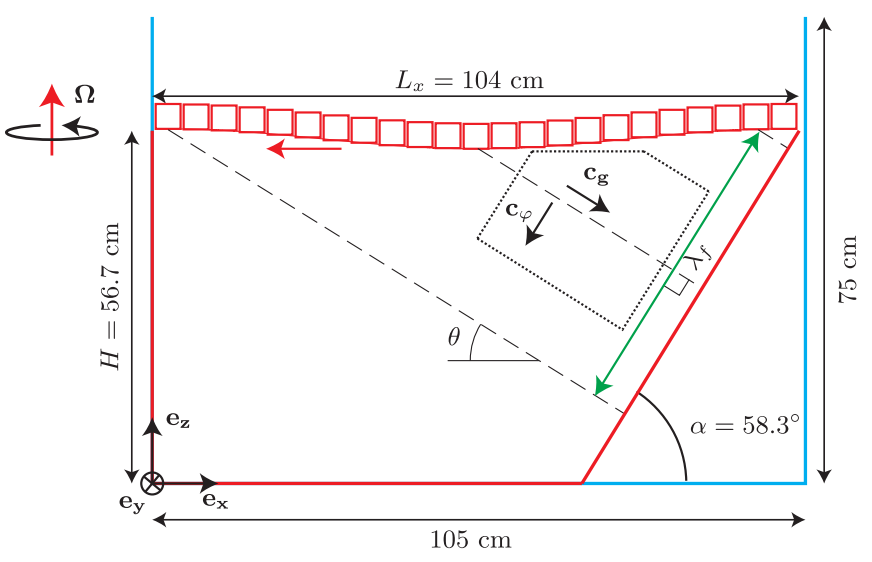

FIG. 1. Scheme of the water tank (in blue) containing the trapezoidal cavity (in red). The upper wall of the cavity is made of a series of $n=23$ horizontal bars, each of them connected to a linear motor able to drive it in a vertical motion. The truncated dashed rectangle shows the region over which the velocity amplitude reported in Fig. 3 is averaged.

allows us to validate scaling laws for the attractor in the nonlinear regime which have been theoretically established assuming that the linear attractor model is still valid in the nonlinear regime if one replaces the fluid viscosity by a turbulent viscosity. We further report a detailed study of the subharmonic waves produced by the triadic resonance instability responsible for the attractor nonlinear evolution mentioned above. We show that, as the forcing Reynolds number is increased, the two typical frequencies, at which subharmonic waves are produced, are moving away from half the attractor frequency, in contradiction with what is expected from the theory of the triadic resonance instability as well as from numerical simulations of inertial wave attractors [36]. We suggest that these differences could be related to the three dimensionality of fluid motions in inertial waves interacting here with the cavity vertical walls. We finally show that a significant proportion of the waves produced by the instability are three dimensional, breaking the invariance in one horizontal direction of the forcing and of the attractor, which could also be a consequence of the wave interaction with the vertical walls.

\section{EXPERIMENTAL SETUP}

The flow is generated in a trapezoidal cavity of height $H=56.7 \mathrm{~cm}$, length $L_{x}=104 \mathrm{~cm}$, and width $L_{y}=105 \mathrm{~cm}$ as illustrated in Fig. 1. This cavity is contained in a parallelepipedic tank of $105 \times 105 \mathrm{~cm}^{2}$ base and $75 \mathrm{~cm}$ height filled with $63 \mathrm{~cm}$ of water. One wall of the cavity is a plate tilted by an angle $\alpha=58.3^{\circ}$ with respect to the horizontal. The forcing of the flow is realized by the upper wall of the cavity which is made of a series of 23 horizontal bars, $86 \mathrm{~cm}$ long and centered in the tank in the $y$ direction. The bars have a square section of $40-\mathrm{mm}$ sides in the $(x, z)$ plane and are spaced of $5 \mathrm{~mm}$ in the $x$ direction. Each of these bars is connected to a linear motor able to drive it in a vertical motion. This wave maker imposes the upper cover of the flow to approximate the wavy shape

$$
Z(x, t)=H+A\left[\cos \left(\sigma_{0} t+k_{0} x\right)-1\right],
$$

where $k_{0}=2 \pi / \lambda_{0}$ and two values of the wavelength have been considered: $\lambda_{0}=11 \times 4.5 \mathrm{~cm}=$ $49.5 \mathrm{~cm}$ and $\lambda_{0}=22 \times 4.5 \mathrm{~cm}=99 \mathrm{~cm}(4.5 \mathrm{~cm}$ is the size of an oscillating bar plus the interval between two bars). The whole system is mounted on a 2-m-diam platform rotating at a constant rate of $\Omega=3$ or $18 \mathrm{rpm}$ about the vertical axis $z$. The rotation of the platform is set at least $30 \mathrm{~min}$ before the wave maker is started to avoid transient spin-up recirculations. The angular frequency of 


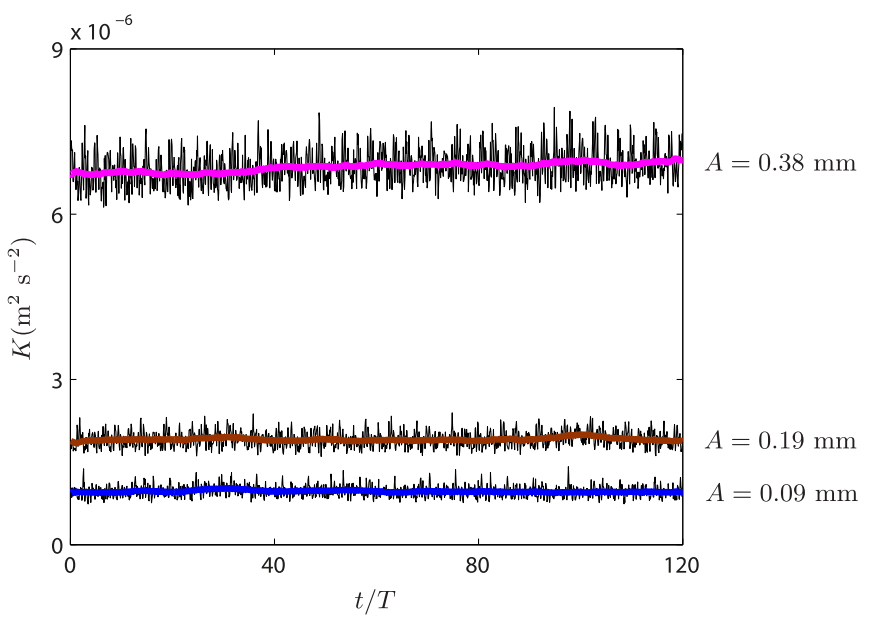

FIG. 2. Time series of the spatially averaged kinetic energy $K=\left\langle u_{x}^{2}+u_{z}^{2}\right\rangle / 2$ for $\Omega=18 \mathrm{rpm}, \lambda_{f}=$ $52.4 \mathrm{~cm}$, and three forcing amplitudes $A=0.09,0.19$, and $0.38 \mathrm{~mm}$. Time $t$ is normalized by the period $T=2 \pi / \sigma_{0}$ of the wave maker. For each time series the black thin line shows the original data and the thick line its moving average using a time window of $5 T$.

the wave maker is set to $\sigma_{0}=0.85 \times 2 \Omega$. The amplitude $A$ of the bar motion $Z(x, t)$ is varied in the range [0.09 $\mathrm{mm}, 18 \mathrm{~mm}]$.

The two components $\left(u_{x}, u_{z}\right)$ of the velocity field are measured in the vertical plane $y=y_{0}=$ $L_{y} / 3$ using a particle image velocimetry (PIV) system mounted in the rotating frame $(y=0$ is the front side of the tank). The fluid is seeded with $10-\mu \mathrm{m}$ tracer particles and illuminated by a laser sheet generated by a corotating $140-\mathrm{mJ}$ Nd:YAG pulsed laser. Pairs of images of particles are acquired using two $2360 \times 1776$ pixel cameras. Using a spatial calibration, the two images in each pair are combined into a single image covering the whole trapezoidal cavity. For wave-maker amplitudes $A \leqslant 1.50 \mathrm{~mm}$, image acquisition consists of series of 1440-5760 image pairs recorded at a rate between 1.5 and $24.4 \mathrm{~Hz}$ depending on the wave-maker amplitude $A$ and on the rotation rate $\Omega=3$ or $18 \mathrm{rpm}$. These values correspond to the acquisition of 120 periods of the wave maker with a time resolution between 12 and 48 image pairs per wave-maker period. For the wave-maker amplitudes $A$ larger than $1.5 \mathrm{~mm}$, acquisitions consist in the regular recording of two pairs of images separated by a time interval $d t \in[9 \mathrm{~ms}, 29 \mathrm{~ms}]$. This double-frame PIV configuration is rendered necessary by the large amplitude of the fluid velocity. For these large values of $A, 120-360$ periods of the wave maker are recorded with a time resolution of 12 doublets of image pairs per wavemaker period. We finally compute cross correlation between successive images over windows of $32 \times 32$ pixels with $50 \%$ overlap. This produces velocity fields of spatial resolution $4.17 \mathrm{~mm}$, with 130 lines of between 164 (at the bottom) and 244 (at the top) vectors almost covering the whole section of the cavity. A few acquisitions have been realized during the transient settling of the flow after the wave maker is started. They have revealed that a steady state is reached after a few dozen periods of the wave-maker forcing. In the data discussed in the following, image acquisition is started after 500 periods of the wave-maker oscillation. In order to illustrate the degree of statistical stationarity of the flow, we report in Fig. 2 the time series of the kinetic energy $K=\left\langle u_{x}^{2}+u_{z}^{2}\right\rangle / 2$ for three experiments, the angular brackets denoting the spatial average over the measurement plane.

In an unbounded domain, our wave maker is expected to generate an inertial wave with its energy propagating at an angle $\theta=\cos ^{-1}\left(\sigma_{0} / 2 \Omega\right) \simeq 32.0^{\circ}$ with respect to the horizontal and of wavelength $\lambda_{f}=\lambda_{0} \sin \theta=26.2$ or $52.4 \mathrm{~cm}$ (see Fig. 1). In line with Eq. (2), the phase of the wave maker propagates toward decreasing values of $x$, selecting the excitation of a wave with an energy propagating toward increasing $x$ (see the group $\mathbf{c}_{\mathbf{g}}$ and phase $\mathbf{c}_{\varphi}$ velocities in Fig. 1). A more subtle 


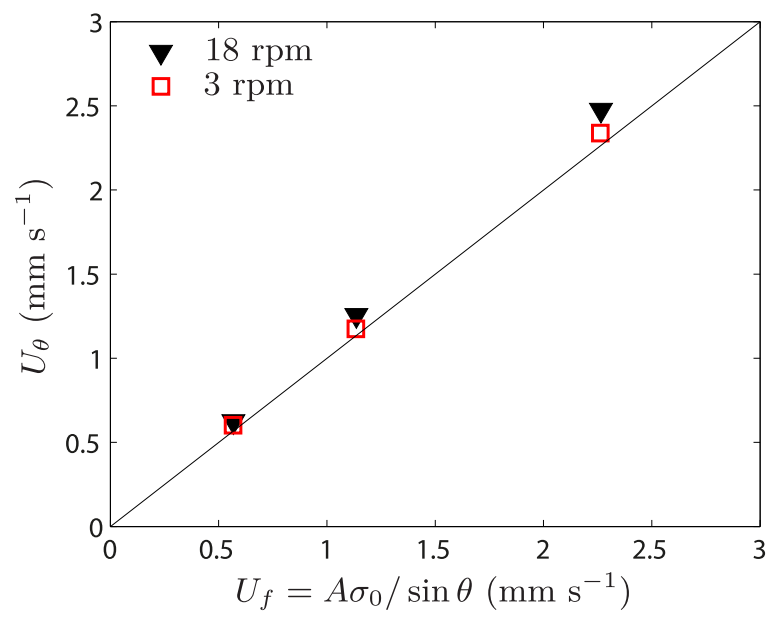

FIG. 3. Velocity amplitude $U_{\theta}$ of the wave excited by the generator in the cavity in the absence of the tilted plane for $\Omega=3$ and $18 \mathrm{rpm}, \lambda_{f}=52.4 \mathrm{~cm}$, and three forcing amplitudes $A=0.09,0.19$, and $0.36 \mathrm{~mm}$. The data are reported as a function of $U_{f}=A \sigma_{0} / \sin \theta$. The solid line indicates the identity function.

point is to predict the velocity amplitude of the raw wave excited by the wave maker. Considering the small thickness of the Ekman boundary layers expected on the wave maker, $\delta=\sqrt{v / 2 \Omega} \simeq 0.5 \mathrm{~mm}$, one can assume that an effective free-slip condition holds at the scale of the wave [39]. One can therefore expect that the wave maker prescribes the $z$ component of the velocity field. Since in an inertial wave fluid particles describe anticyclonic circular translation in planes tilted by the angle $\theta$ $[1,8,39]$, the amplitude of the wave velocity components (in the $\theta$ and $y$ directions) are expected to be $U_{f}=A \sigma_{0} / \sin \theta \in[0.6,36.2] \mathrm{mm} \mathrm{s}^{-1}$. This prediction is expected to hold when $\theta \rightarrow 90^{\circ}$, but probably fails when $\theta \rightarrow 0^{\circ}$ since it would imply a divergence of the wave velocity amplitude.

To confirm that this prediction $U_{f}=A \sigma_{0} / \sin \theta$ holds at the angle $\theta \simeq 32.0^{\circ}$ used in the experiments, we have realized a few complementary experiments without the tilted plane. In Fig. 3 we report the velocity oscillation amplitude $U_{\theta}$ in the $\theta$ direction, i.e., the direction of the wave group velocity, as a function of the predicted amplitude $U_{f}=A \sigma_{0} / \sin \theta$. To get $U_{\theta}$, we first Fourier filter the velocity field obtained from particle image velocimetry at frequency $\sigma_{0}$. We then compute the amplitude of the velocity oscillations in the $\theta$ direction and finally take the spatial average of the resulting field over the region delineated by the dashed lines in Fig. 1 in the center of the excited wave. Figure 3 confirms that the excited wave amplitude is indeed close to $U_{f}=A \sigma_{0} / \sin \theta$ within a $10 \%$ precision which confirms the relevance of our estimate. The values found for $U_{\theta}$ are actually slightly larger than $U_{f}$. This discrepancy can be a consequence of the fact that the assumptions made to model $U_{f}$, i.e., the free-slip boundary condition and the fact that the wavemaker is watertight (which is not the case because of the 5-mm gap between the bars), are only valid to the first order. This discrepancy might also reveal the contribution to the measured velocity amplitude $U_{\theta}$ of the wave after its reflections on the four cavity walls which is superimposed on the original wave. In the following, $U_{f}$ and $\lambda_{f}$ will be referred to as the forcing velocity and forcing wavelength and $\operatorname{Re}_{f}=U_{f} \lambda_{f} / v=A \sigma_{0} \lambda_{0} / v$ the forcing Reynolds number.

\section{WAVE ATTRACTOR IN THE LINEAR REGIME AND BEYOND}

Attractors of internal waves of inertia or gravity can emerge in cavities with walls nonparallel or non-normal to the rotation or stratification axis. This is due to the anomalous reflection laws of these waves which keep constant their propagation angle $\theta$ with respect to the horizontal during a reflection [25]. As a consequence, when reflecting downward on a wall tilted by an angle $\alpha$ 


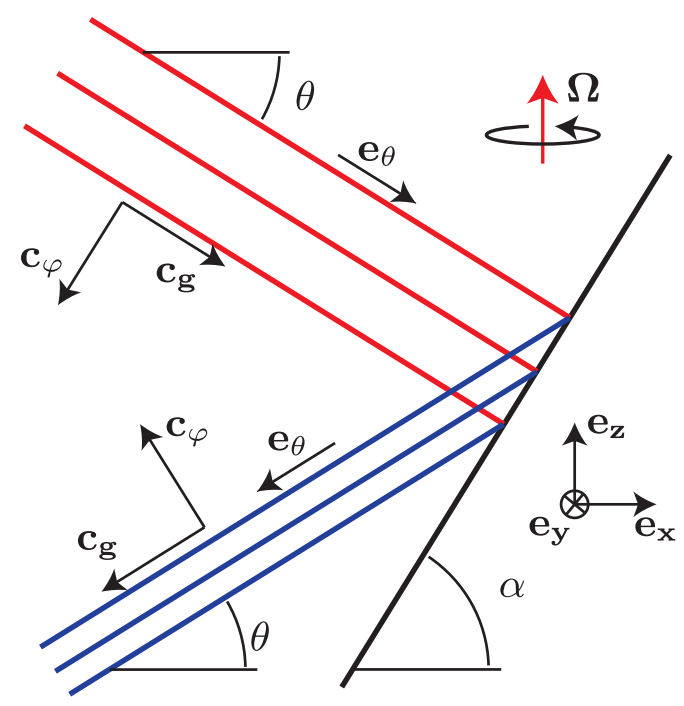

FIG. 4. Reflection of an inertial wave of angular frequency $\sigma=2 \Omega \cos \theta$ on a plane tilted by an angle $\alpha$. The vectors $\mathbf{c}_{\varphi}$ and $\mathbf{c}_{\mathrm{g}}$ show the directions of the phase and group velocities for the incident and reflected beams.

(see Fig. 4), a wave has its transverse length scales reduced by a factor

$$
\gamma=\frac{\sin (\alpha+\theta)}{\sin (\alpha-\theta)}
$$

which is equal to 2.25 here in the experiments. Moreover, noting that the group velocity of a plane inertial wave of angular frequency $\sigma=2 \Omega \cos \theta$ has a magnitude $\left|\mathbf{c}_{\mathbf{g}}\right|=\Omega \lambda \sin \theta / \pi$, the conservation of the energy flux implies that $U_{\theta} \lambda$ and $U_{y} \lambda$ are conserved at reflection (at least in the inviscid case [40]), $\lambda$ being the wavelength and $U_{\theta}$ and $U_{y}$ the amplitude of the velocity oscillations in the $\mathbf{e}_{\theta}$ and $\mathbf{e}_{\mathbf{y}}$ directions, respectively (see Fig. 4). In addition, the wave vorticity scaling as $U_{y, \theta} / \lambda$ is amplified by a factor $\gamma^{2}$ at the reflection.

In a closed domain, this focusing process leads, for certain geometry-dependent ranges of angles $\theta$, to an energy concentration on a wave attractor [26-29]. In the trapezoidal cavity considered here, a $(1,1)$ inertial wave attractor is expected when waves are generated with a propagation angle $\theta$ in the range between

$$
\theta_{1}=\tan ^{-1}\left(H / L_{x}\right) \simeq 28.6^{\circ}
$$

and

$$
\theta_{2}=\tan ^{-1}\left(\frac{H \tan (\alpha)}{L_{x} \tan (\alpha)-H}\right) \simeq 39.4^{\circ} .
$$

The notation $(1,1)$ corresponds to the simplest class of an attractor in a trapezoidal cavity and follows from the nomenclature introduced in [27], the first number being the number of reflections on the bottom wall and the second the number of reflections on the sloping wall. The two limit angles $\theta_{1}$ and $\theta_{2}$ correspond to the slope of the diagonals of the trapezoidal cavity.

In Fig. 5 we show for the angle $\theta \simeq 32.0^{\circ}$ used in the experiments the unique closed parallelogram with its vertices on the walls of the cavity and its sides all tilted by an angle $\theta$. This parallelogram corresponds to the inviscid wave attractor: Ray tracing for any wave at $\sigma_{0}=2 \Omega \cos \theta$ will converge toward this parallelogram. The rate of convergence of the rays toward the inviscid attractor in a trapezoidal cavity has been characterized by Maas et al. in [27] via the calculation of the Lyapunov exponents for each couple of nondimensional geometric parameters 


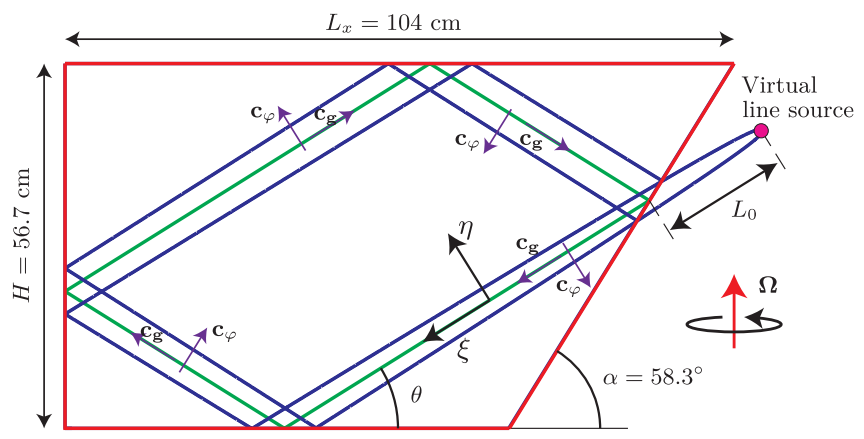

FIG. 5. Scheme of the theoretical inviscid (in green) and viscous (in blue) attractors in the trapezoidal cavity, for $\theta=32.0^{\circ}$. The blue lines more precisely show the width at midheight of the velocity envelope of the Moore-Saffman self-similar wave beam [Eq. (6)]. The vectors $\mathbf{c}_{\varphi}$ and $\mathbf{c}_{\mathbf{g}}$ show for each attractor branch the direction of the phase and group velocities.

$\left(d=1-2 H /\left(L_{x} \tan \alpha\right), \tau=2 H /\left(L_{x} \tan \theta\right)\right)$. In [27], internal gravity waves are considered, but since inertial waves behave exactly the same way regarding two-dimensional ray tracing, the Lyapunov exponents diagram as a function of $(d, \tau)$ is expected to be identical in our case. This diagram reveals regions of strong convergence called Arnold tongues [27,41]. In our experiments, one has $(d \simeq 0.33, \tau \simeq 1.74)$, which falls in the middle of the Arnold tongue corresponding to the $(1,1)$ attractor.

In a viscous fluid, the inviscid concentration of energy on a line attractor is prevented by viscous dissipation of waves during their propagation. The structure of the attractor can eventually be seen as the result of a balance between focusing at the sloping wall and the viscous spreading of a self-similar polychromatic wave beam [28,33-35]. As proposed in [35] for internal wave attractors in a linearly stratified fluid, the four branches of the attractor, once unwrapped, can indeed be seen as part of a beam emitted by a virtual line source (invariant in the $y$ direction), located at a distance $L_{0}$ upstream of the focusing reflection (see Fig. 5).

From a general point of view, the wave beam excited by a line source has a self-similar transverse structure. Its velocity component in the propagation direction $\theta$ is given by (see Ref. [6] for details)

$$
u_{\xi}^{(m)}(\xi, \eta, t)=U_{0}\left(\frac{\ell}{\xi}\right)^{(m+1) / 3}\left[c_{m}(\eta / \delta) \cos (\sigma t+\phi)+s_{m}(\eta / \delta) \sin (\sigma t+\phi)\right]
$$

and the out-of-plane vorticity component by

$$
\omega_{y}^{(m)}(\xi, \eta, t)=\frac{U_{0}}{\ell}\left(\frac{\ell}{\xi}\right)^{(m+2) / 3}\left[-s_{m+1}(\eta / \delta) \cos (\sigma t+\phi)+c_{m+1}(\eta / \delta) \sin (\sigma t+\phi)\right],
$$

where $\xi$ is the distance from the source, $\eta$ the local transverse coordinate,

$$
\ell=\frac{v^{1 / 2}}{\left[(2 \Omega)^{2}-\sigma_{0}^{2}\right]^{1 / 4}}
$$

a viscous scale, and

$$
\delta(\xi)=\xi^{1 / 3} \ell^{2 / 3}
$$

the scaling law followed by the width (and all other transverse length scales) of the beam. In the experiments, $\ell \simeq 1.74 \mathrm{~mm}$ for $\Omega=3 \mathrm{rpm}$ and $\ell \simeq 0.71 \mathrm{~mm}$ for $\Omega=18 \mathrm{rpm}$. The functions $c_{m}$ and $s_{m}$ have been introduced by Moore and Saffman [42] and Thomas and Stevenson [43] to describe self-similar wave beams of inertial and internal waves, respectively. These real functions are 
defined by

$$
c_{m}(\zeta)+i s_{m}(\zeta)=\int_{0}^{\infty} K^{m} e^{-K^{3}+i K \zeta} d K .
$$

In Eqs. (6), (7), and (10), the integer $m+1$ corresponds to the multipolar order of the line source of waves as discussed in [6]: $m=0$ corresponds to a monopolar source and $m=1$ to a dipolar source. Our wave maker produces a large-scale wave with a zero instantaneous net mass flux (because of an integer number of wavelengths) which suggests consideration of the dipolar case $m=1$. However, it is worth noting that Jouve and Ogilvie [36] consider the case $m=0$ in their work, which leads to a successful description of the spatial dependence of the attractor amplitude in their numerical simulations. The multipolar order of the virtual source to be considered here is therefore an open question.

Denoting $L_{a}$ the length of the unwrapped inviscid attractor and $L_{0}$ the distance between the virtual source and the focusing point, the balance between the viscous spreading of the wave between $\xi=L_{0}$ and $\xi=L_{0}+L_{a}$ with length scales increasing as $\xi^{1 / 3} \ell^{2 / 3}$ and the focusing reflection leads to the compatibility relation $L_{0}=L_{a} /\left(\gamma^{3}-1\right)$. In the experiments, the attractor length $L_{a}$ is $214.2 \mathrm{~cm}$ such that $L_{0} \simeq 20.5 \mathrm{~cm}$ (see Fig. 5). This eventually leads to the relation for the transverse length scale of the attractor as a function of along-attractor coordinate $s[35,36,38]$,

$$
\frac{\delta}{L_{a}}=\left(\frac{\ell}{L_{a}}\right)^{2 / 3}\left(\frac{s}{L_{a}}+\frac{1}{\gamma^{3}-1}\right)^{1 / 3} .
$$

Here $s=\xi-L_{0}$ is the distance along the unwrapped attractor starting from the focusing reflection.

The relevance of the scaling law (11) was tested numerically for gravity waves in a stratified fluid by Grisouard et al. [35], who indeed observed power-law behaviors but with exponents departing from $1 / 3$ by $-25 \%$ and $+40 \%$ in the two reported configurations. In numerical simulations of a rotating tilted square, Jouve and Ogilvie [36] confirmed more clearly the predicted scaling laws for the attractor width $(\xi / \ell)^{1 / 3}$ and the velocity amplitude $(\xi / \ell)^{-1 / 3}$ (case $m=0$ ) with the position along the attractor as well as the relevance of the Moore-Saffman transverse structure of the beam. From an experimental point of view, Brouzet et al. [38] studied the evolution with time of the wavelength in the attractor during its transient growth and decay phases in a linearly stratified fluid. These data revealed a decrease toward a steady-state value during the forced growth and a further decrease during the free decay. Comparable results were previously reported by Hazewinkel et al. [34].

Jouve and Ogilvie [36] also studied the nonlinear regime of the attractor of inertial waves and showed the emergence of a local instability close to each focusing point. This instability transfers the energy of the wave attractor toward two subharmonic waves with their frequencies in triadic resonance with the primary wave frequency. This scenario is consistent with the one reported in internal gravity wave attractor experiments by Scolan et al. [37]. These local subharmonic instabilities of an internal wave attractor are actually very similar to instabilities of plane-wave beams observed experimentally in rotating [8] and stratified [44] fluids. In this context, it has been shown $[45,46]$ that one should take into account the finite width of the wave beam, i.e., the small number of wavelengths contained in the transverse extension of the wave attractor (typically 1), in order to correctly predict the growth rate of the secondary waves generated by the instability.

In this context, one can highlight a remarkable feature of the instability of the experimental plane inertial wave reported by Bordes et al. [8] compared to all other mentioned instabilities: In the temporal spectrum, two wide bumps are observed, centered around two frequencies in triadic resonance with the primary wave frequency. This is in strong contrast with the internal gravity waves experiments with either a plane wave [44] or an attractor [37], but also with the inertial wave attractor simulations [36] for which the instability is very selective in terms of secondary wave frequencies. The physical origin of this specificity of experimental inertial waves has been unclear up to now. 


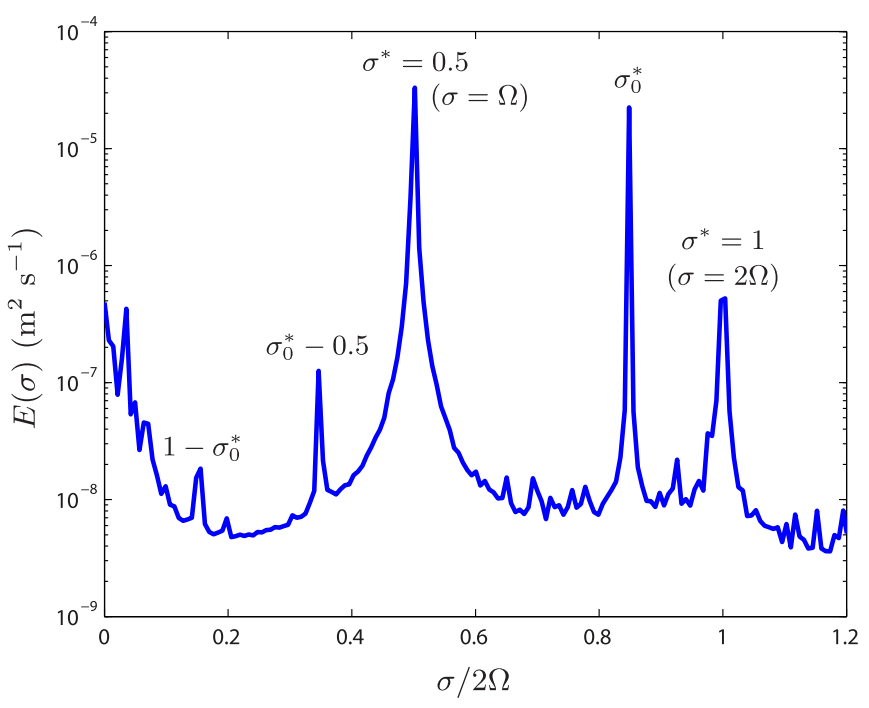

FIG. 6. Temporal power spectral density $E(\sigma)$ [Eq. (12)] as a function of the normalized frequency $\sigma^{*}=$ $\sigma / 2 \Omega$ for $A=0.09 \mathrm{~mm}, \lambda_{f}=52.4 \mathrm{~cm}$, and $\Omega=18 \mathrm{rpm}$. We have highlighted five peaks at frequencies $\sigma_{0}^{*}=$ $\sigma_{0} / 2 \Omega, \sigma^{*}=0.5$ (i.e., $\sigma=\Omega$ ), $\sigma^{*}=1$ (i.e., $\sigma=2 \Omega$ ), $\sigma^{*}=\sigma_{0}^{*}-0.5$, and $\sigma^{*}=1-\sigma_{0}^{*}$.

Finally, in [36,38] in which subharmonic triadic instabilities of numerical inertial and experimental internal wave attractors are reported, a thickening of the attractor beam is reported, as the forcing amplitude is increased. This thickening can be understood qualitatively as the consequence of the extraction of energy from the attractor at frequency $\sigma_{0}$ by the triadic instability which acts as an effective turbulent dissipation: The instability then naturally produces an attractor with a smaller relative amplitude and larger transverse scales as the forcing amplitude is increased.

\section{EXPERIMENTAL RESULTS}

\section{A. Linear regime}

In our experiments, energy is injected at frequency $\sigma_{0}=0.85 \times 2 \Omega$ by the wave generator. In order to uncover the frequency content of the flow, we compute for each experiment the temporal power spectral density of the velocity field as

$$
E(\sigma)=\frac{4 \pi}{T}\left\langle\left|\tilde{u}_{j}(x, z, \sigma)\right|^{2}\right\rangle,
$$

where

$$
\tilde{u}_{j}(x, z, \sigma)=\frac{1}{2 \pi} \int_{0}^{T} u_{j}\left(x, y_{0}, z, t\right) e^{-i \sigma t} d t
$$

is the temporal Fourier transform of $u_{j}\left(x, y_{0}, z, t\right), j=x, z$, the angular brackets denote the spatial average over the measurement plane, $T$ is the acquisition duration, and $y_{0}=L_{y} / 3$.

In Fig. 6 we report the temporal energy spectrum $E(\sigma)$ for the forcing wavelength $\lambda_{f}=52.4 \mathrm{~cm}$ and the lowest forcing amplitude $A=0.09 \mathrm{~mm}$ at $\Omega=18 \mathrm{rpm}$ as a function of the normalized frequency $\sigma^{*}=\sigma / 2 \Omega$. This spectrum is mainly composed of a sharp peak at the forcing frequency $\sigma_{0}$ as well as a secondary but energetic peak at the frequency of the rotating platform $\sigma=\Omega$. The latter corresponds to a flow excited by the earth's rotation which induces a precession of the rotating platform (see Refs. [13,47] and references therein). In the case of a spherical or ellipsoidal cavity under precession, it is known as the tilt-over flow and has been extensively 

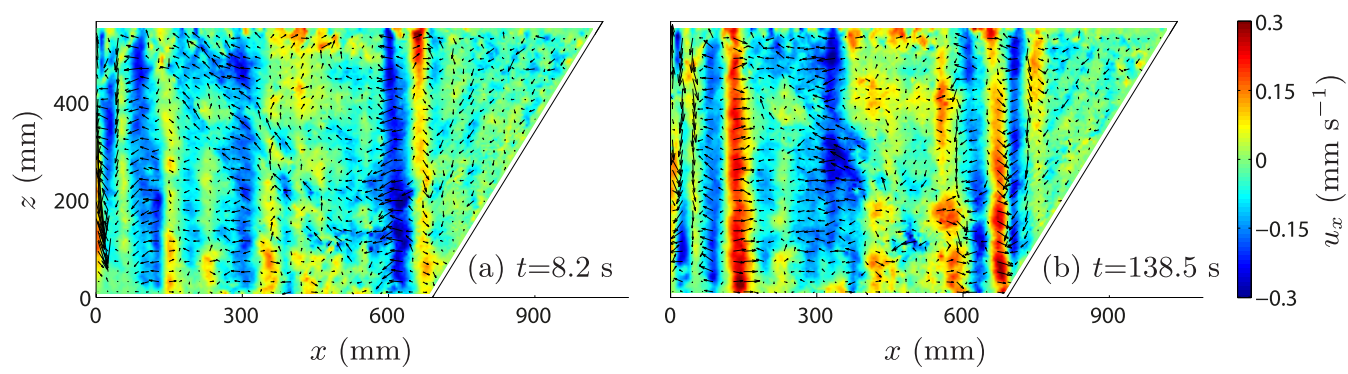

FIG. 7. Snapshots of the velocity field after a temporal moving average over a time window of eight wavemaker periods (i.e., a cutoff frequency of $\sigma^{*} \simeq 0.11$ ) in the vertical plane $y=y_{0}=L_{y} / 3$ for $\lambda_{f}=52.4 \mathrm{~cm}$ and the lowest forcing amplitude $A=0.09 \mathrm{~mm}$ at $\Omega=18 \mathrm{rpm}$.

studied due to its relevance in astrophysics [48]. One can also note the presence of energy at low frequencies $\sigma / 2 \Omega<0.15$. In a previous work using the same rotating platform [8], it was shown that the energy peak at zero frequency (with a tail extending up to $\sigma^{*} \simeq 0.15$ ) was already present when the flow forcing is off, suggesting that the low-frequency spectral component is possibly the consequence of thermal convection in the water tank. The observation of the velocity field temporally smoothed over a large timescale actually revealed the presence of columns which are slowly drifting in the water tank, resembling the columns observed in rotating thermal convection experiments [49,50]. Here we observe the same kind of vertical columns, dominated by horizontal velocities, in the low-pass frequency filtered velocity field (two snapshots are shown in Fig. 7) which could possibly be the consequence of thermal convection. Nevertheless, even if no clear signs are observed here, one should leave open the possibility that part of the energy present at very low frequencies in our experiments could be related to nonlinearities, such as steady streaming [51,52] or Stokes drift [53], affecting the flow motions at the forcing frequency $\sigma_{0}$ or at the platform frequency $\Omega$. Other weakly energetic peaks are also present in the spectrum corresponding to the first harmonic of the tilt-over flow $\left(\sigma^{*}=1\right)$, to interactions between the forcing and the tilt-over flow at $\sigma^{*}=\sigma_{0}^{*}-0.5 \simeq 0.35$, and to interactions between the forcing and the first harmonic of the tilt-over flow at $\sigma^{*}=1-\sigma_{0}^{*} \simeq 0.15$.

Overall, the flow produced by the wave generator with the lowest forcing amplitude at $\Omega=$ $18 \mathrm{rpm}$ seems to be in the linear regime. In order to discard the tilt-over and the low-frequency flow components, we Fourier filter the velocity field at the forcing frequency $\sigma_{0}$. Figure 8 shows a snapshot of the corresponding field on which the width at midamplitude of the theoretical attractor (6) is superimposed. The velocity field reveals a concentration of energy along the theoretical attractor, in good agreement with the theory. This concentration is however only partial since one can see other wave beams tilted by the angle $\theta$ outside the region where the theoretical attractor is expected: In Fig. 8 the velocity magnitude in the attractor beam (from 1 to $2 \mathrm{~mm} / \mathrm{s}$ ) is actually only 3-7 times larger than the forcing velocity magnitude $U_{f} \simeq 0.27 \mathrm{~mm} / \mathrm{s}$. As a consequence, since the wave maker injects energy over the whole width of the water tank, we naturally find wave beams with a non-negligible amplitude outside the attractor region.

To further compare the experimental flow at frequency $\sigma_{0}$ and the theoretical attractor, we study its transverse profile as a function of the longitudinal coordinate $s=\xi-L_{0}$ along the inviscid attractor. To do so, we notice that each of the four branches of the attractor has its wave vector in a different quadrant of the wave-vector plane $\left(k_{x}, k_{z}\right)$ (the wave vector is aligned with the phase velocity $\mathbf{c}_{\varphi}$; see Fig. 5). We perform a Hilbert filtering of the velocity field (see Ref. [54] for details). It consists in computing the temporal Fourier transform of the raw velocity field, band-pass filtering the result around the frequency of interest $\sigma_{0}$ (keeping only positive frequencies), and computing the inverse Fourier transform. We then take the two-dimensional (2D) spatial Fourier transform of the resulting complex field relative to $x$ and $z$, set to zero the values of the resulting field except in the wave-vector quadrant of interest, and finally compute the inverse 2D Fourier transform in 


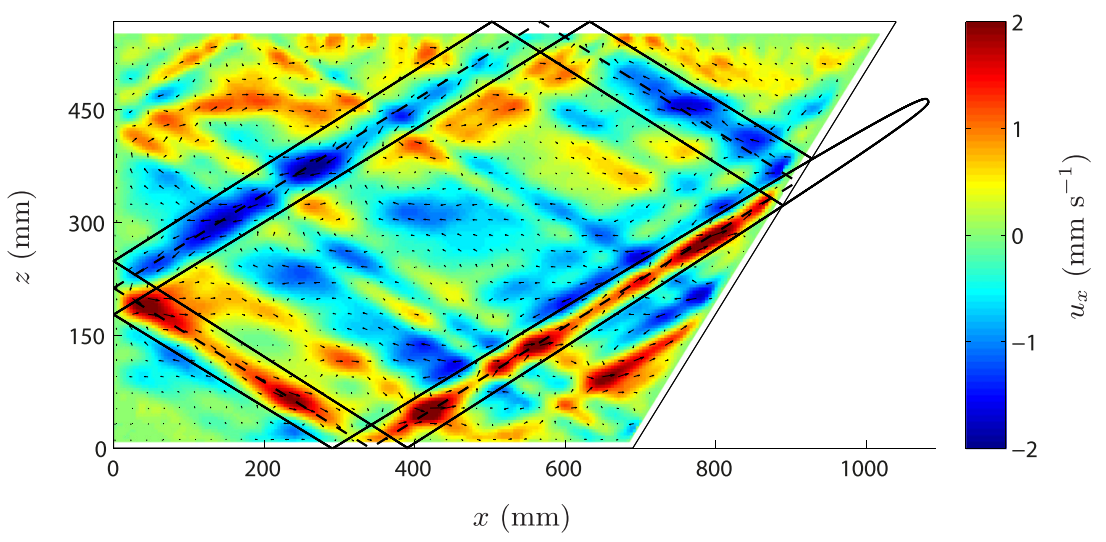

FIG. 8. Attractor in the linear regime: snapshot of the velocity field in the vertical plane $y=y_{0}=L_{y} / 3$ Fourier filtered at the forcing frequency $\sigma_{0}$ for $\lambda_{f}=52.4 \mathrm{~cm}$ and the lowest forcing amplitude $A=0.09 \mathrm{~mm}$ at $\Omega=18 \mathrm{rpm}$. A sketch of the theoretical attractor is superimposed on the experimental field: The dashed line shows the inviscid skeleton and the two solid lines delineate the width at midheight of the viscous beam longitudinal velocity amplitude [Eq. (6)].

space. Taking twice the real part of the result eventually provides the velocity field of the waves at frequency $\sigma_{0}$ and with their wave vector in a given quadrant [54]. We finally perform a temporal phase average at $\sigma_{0} .{ }^{1}$ As an illustration, we report in Fig. 9 a snapshot of the field resulting from the Hilbert filtering; this snapshot is divided in four regions, each corresponding to a given theoretical attractor branch and to a Hilbert filtering selecting the wave-vector quadrant of the theoretical attractor branch. One can note the presence of a few wave beams outside the theoretical attractor which reveals that the focusing of the energy injected by the forcing in the attractor, although clear, is only partial.

In the inset of Fig. 9 we report a transverse profile (along $\eta$ ) of the out-of-plane vorticity component $\omega_{y}\left(\xi=s+L_{0}, \eta, \varphi\right)$ corresponding to the $\sigma_{0}$ and $\left(k_{x}>0, k_{z}<0\right)$ Hilbert filtered velocity field. This transverse profile is taken at the coordinate $s=49.7 \mathrm{~cm}$ along the attractor axis (corresponding to the solid line in Fig. 9) at a given arbitrary phase, still for $\lambda_{f}=52.4 \mathrm{~cm}$ and the lowest forcing amplitude $A=0.09 \mathrm{~mm}$ at $\Omega=18 \mathrm{rpm}$. We also report the corresponding experimental wave beam envelope $\omega_{y, 0}(\xi, \eta)=\sqrt{2\left\langle\omega_{y}(\xi, \eta, \varphi)^{2}\right\rangle_{\varphi}}$, where \langle\rangle$_{\varphi}$ stands for the average over the phase $\varphi \in[0,2 \pi]$. From such curves, we measure, as a function of the longitudinal position $\xi$, the beam vorticity amplitude $W(\xi)=\max _{\eta}\left[\omega_{y, 0}(\xi, \eta)\right]$ as well as the wavelength $\lambda(\xi)$ estimated as the mean value over $\varphi$ of twice the transverse distance between the maximum and minimum of the vorticity profile $\omega_{y}(\xi, \eta, \varphi)$.

In Fig. 10 we report the vorticity amplitude $W(\xi)$ for $A=0.09 \mathrm{~mm}, \lambda_{f}=52.4 \mathrm{~cm}$, and $\Omega=$ $18 \mathrm{rpm}$ as a function of coordinate $\xi=s+L_{0}$ along the unwrapped theoretical beam emitted by the virtual source. Data are missing on five portions of $\xi$ corresponding to the regions where the velocity field cannot be measured by PIV close to the reflections on the cavity walls. The attractor amplitude $W(\xi)$ shows significant oscillations that are due to interferences of the wave attractor with the additional inertial waves at $\sigma_{0}$ present in the cavity (see Fig. 8) as well as to interferences between two branches of the attractor close to a reflection. Nevertheless, one can observe good agreement between the data and a power law of exponent $-2 / 3$ in agreement with the scaling predicted by the theory (7) for a monopolar source of waves $m=0$. The observation of this $-2 / 3$ spatial decay exponent is consistent with the numerical data reported by Jouve and Ogilvie [36]. It

\footnotetext{
${ }^{1}$ The phase average of the time series $f(t)$ at frequency $\sigma_{0}$ is defined by $\hat{f}(\varphi)=\sum_{n=1}^{N} f\left((\varphi+n 2 \pi) / \sigma_{0}\right) / N=$ $\sum_{n=1}^{N} f\left(t+n 2 \pi / \sigma_{0}\right) / N$, where $N$ is the total number of period $2 \pi / \sigma_{0}$ of the signal and $\varphi=\sigma_{0} t$.
} 


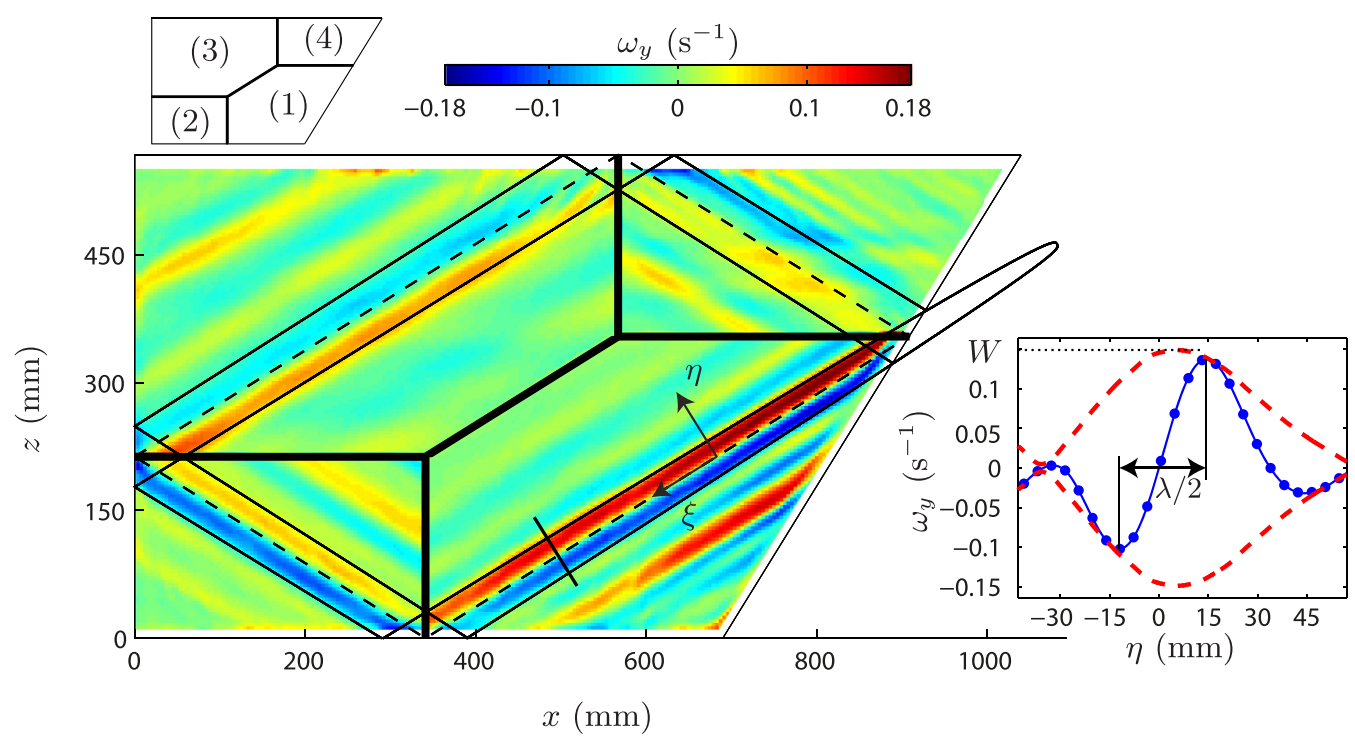

FIG. 9. Attractor in the linear regime: snapshot of the out-of-plane vorticity of the Hilbert filtered field at the forcing frequency $\sigma_{0}$ for $A=0.09 \mathrm{~mm}, \lambda_{f}=52.4 \mathrm{~cm}$, and $\Omega=18 \mathrm{rpm}$. The reported field is actually a combination of four regions in which different wave-vector quadrants have been selected by the Hilbert filtering in agreement with the direction expected for the wave vector in each attractor branch (cf. Fig. 5): In region 1, we keep the wave-vector quadrant $\left(k_{x}>0, k_{z}<0\right)$; in $2,\left(k_{x}>0, k_{z}>0\right)$; in $3,\left(k_{x}<0, k_{z}>0\right)$; and in $4,\left(k_{x}<0, k_{z}<0\right)$. A sketch of the theoretical attractor is superimposed (same layout as in Fig. 8). The inset shows the experimental transverse profile (blue line with data markers) of the $y$ component of the vorticity $\omega_{y}\left(\xi=s+L_{0}, \eta, \varphi\right)$ of the $\sigma_{0}$ and $\left(k_{x}>0, k_{z}<0\right)$ Hilbert-filtered field (region 1). It is taken at coordinate $s=49.7 \mathrm{~cm}$ along the attractor axis (corresponding to the straight line in the snapshot) and at a given arbitrary phase. We also report in the inset the experimental wave-beam envelope (red dashed line) computed from the experimental transverse profiles as $\omega_{y, 0}=\sqrt{2\left\langle\omega_{y}(\xi, \eta, \varphi)^{2}\right\rangle_{\varphi}}$ where \langle\rangle$_{\varphi}$ stands for the average on the phase $\varphi \in[0,2 \pi]$.

shows that the multipolar order of the virtual point source to be considered in the attractor model is $m=0$ (monopolar source) and seems largely independent of the way energy is injected into the system.

In Fig. 11 we show the corresponding evolution with $\xi$ of the wavelength $\lambda$ in the attractor, normalized by the viscous length scale $\ell$ (Eq. 8). As in Fig. 10, data are missing around the reflections on the cavity walls. The excluded ranges of $\xi / \ell$ are larger because estimates of the attractor transverse length scales are prevented when approaching a wall at a distance of the order of these length scales $(\sim 100 \ell-200 \ell)$. We also report in Fig. 11 the theoretical prediction for $\lambda / \ell$ according to Eqs. (7)-(10). We emphasize that this prediction is a power law in $(\xi / \ell)^{1 / 3}$ with a prefactor theoretically prescribed by the Moore-Saffman functions. One sees that, despite the fact that the power-law behavior is not clearly observed in the data, the theory provides correct estimates for $\lambda$. The wavelength is found here to always be slightly larger than the theoretical prediction. Such a tendency is identical to the one reported for experimental gravity waves attractor [38]. In this work as well as in [40], it is proposed that the additional dissipation due to the viscous friction on the vertical walls of the cavity $\left(y=0\right.$ and $\left.y=L_{y}\right)$ leads to an attractor larger than in the 2D theory (invariant in the $y$ direction) by modifying the balance between energy focusing and viscous dissipation. One can finally highlight that in both Figs. 10 and 11 the experimental data in the fourth branch of the attractor are particularly noisy and also significantly depart from the theoretical scaling law. This could be understood by the fact that the fourth branch is the weaker in magnitude 


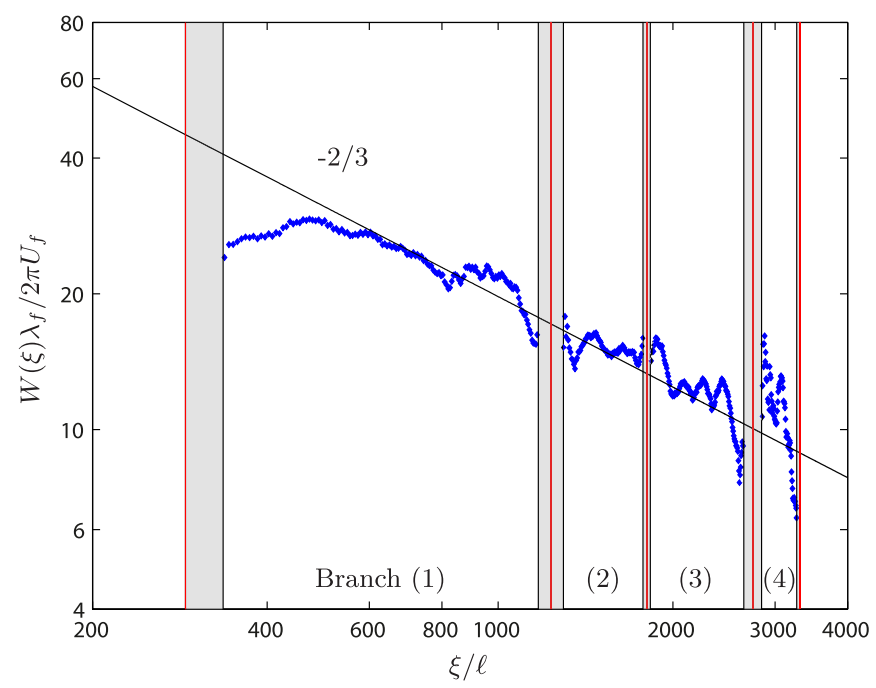

FIG. 10. Vorticity amplitude $W(\xi)$ of the attractor as a function of the coordinate $\xi=s+L_{0}$ for $A=$ $0.09 \mathrm{~mm}, \lambda_{f}=52.4 \mathrm{~cm}$, and $\Omega=18 \mathrm{rpm}$. Here $W(\xi)$ is normalized by the forcing vorticity $2 \pi U_{f} / \lambda_{f}$ and the position $\xi$ by the viscous length scale $\ell$ given by Eq. (8). Vertical red lines show the reflections on the cavity walls and gray regions delineate zones in which PIV measurement is not possible. Following Eq. (7), a power law with an exponent $-2 / 3$ is also shown as a guide for the eyes.

[see Eq. (6)] whereas at the same time it is located where the original wave produced by the wave maker is the strongest. The experimental data in the fourth branch of the attractor are therefore probably strongly affected by interferences between the wave in the attractor and the original wave produced by the wave maker.

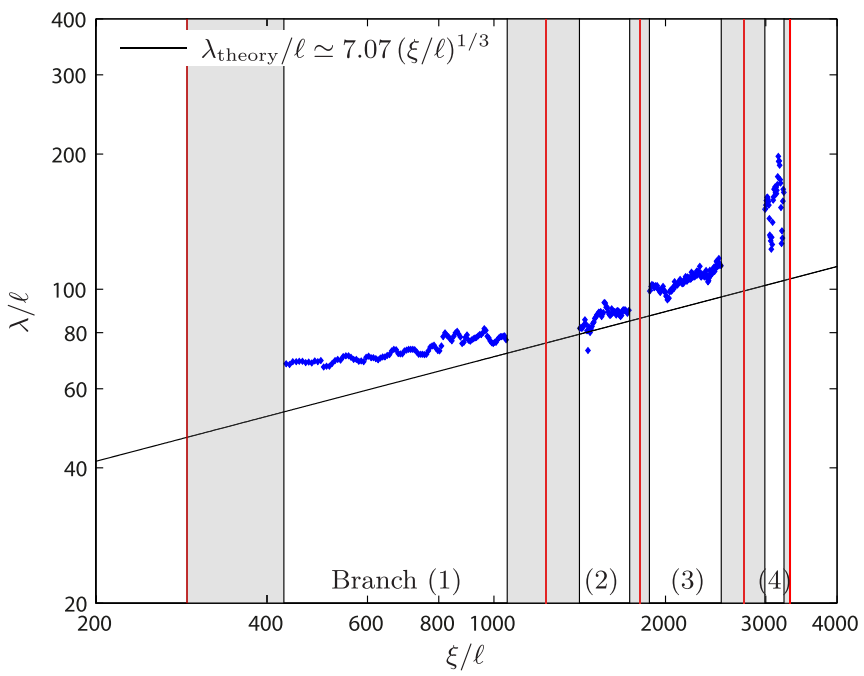

FIG. 11. Normalized wavelength $\lambda / \ell$ in the attractor beam as a function of the normalized distance from the virtual source $\xi / \ell$ for $A=0.09 \mathrm{~mm}, \lambda_{f}=52.4 \mathrm{~cm}$, and $\Omega=18 \mathrm{rpm}$. Vertical red lines show the reflections on the cavity walls and gray regions delineate zones in which boundary effect prevents measurements. The solid line shows the theoretical predictions, in $(\xi / \ell)^{1 / 3}$ and with no adjustable parameter, for the wavelength $\lambda$. 

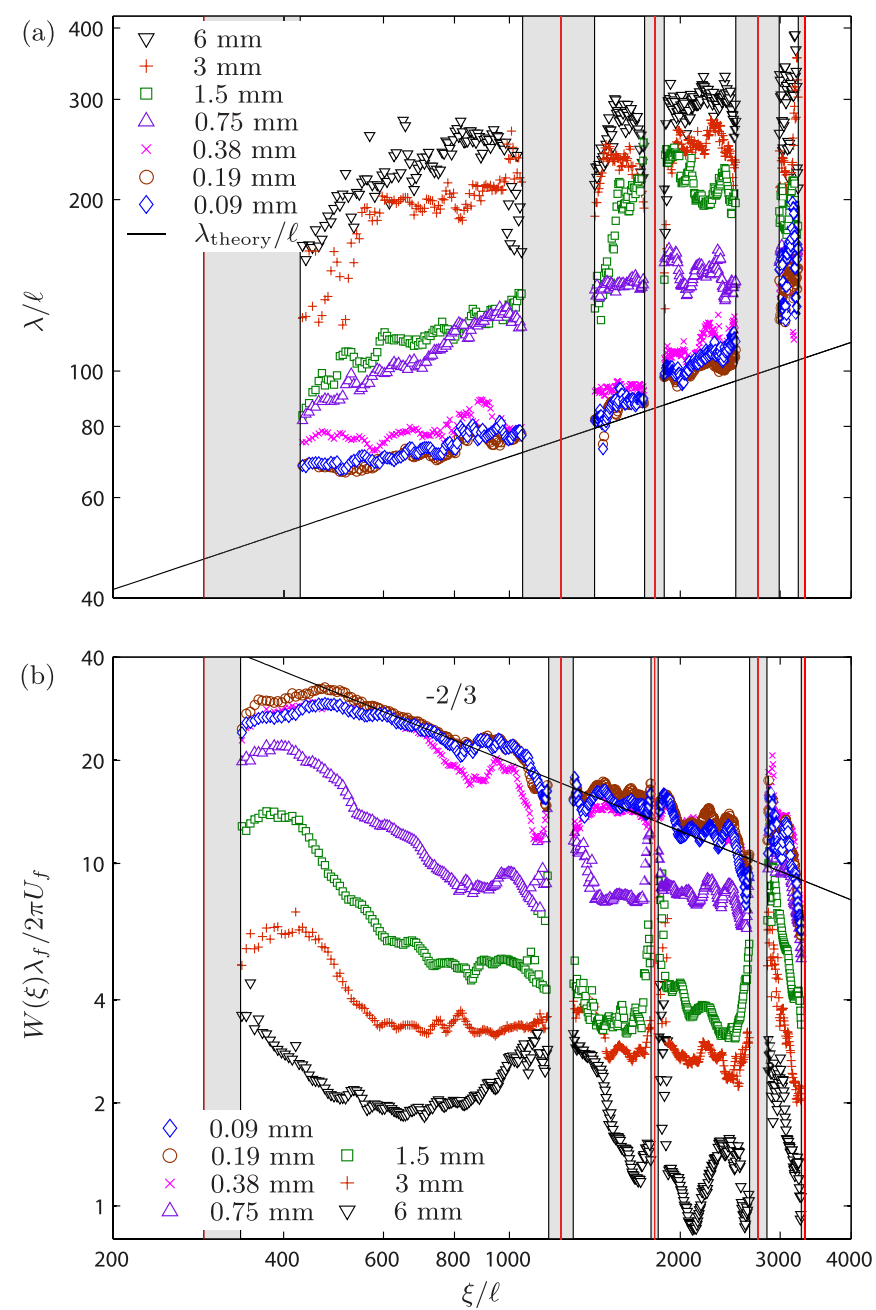

FIG. 12. (a) Normalized wavelength $\lambda / \ell$ and (b) vorticity amplitude $W(\xi) \lambda_{f} / 2 \pi U_{f}$ as a function of the normalized distance from the virtual source $\xi / \ell$ for each forcing amplitude $A$ at $\Omega=18 \mathrm{rpm}$ and $\lambda_{f}=52.4 \mathrm{~cm}$. For both figures, vertical red lines show the reflections of the theoretical attractor on the cavity walls and gray regions zones in which the boundary effect prevents measurements. In (a), the solid line shows the theoretical power law, in $(\xi / \ell)^{1 / 3}$ and with no adjustable parameter, for the linear attractor. In (b), a power law with an exponent $-2 / 3$ is shown, corresponding to the theoretical linear attractor.

\section{B. Nonlinear regime}

We now repeat the previous analysis for increasing forcing amplitude $A$. In Fig. 12 we report the wavelength [Fig. 12(a)] and the out-of-plane vorticity amplitude normalized by the forcing vorticity $2 \pi U_{f} / \lambda_{f}$ [Fig. 12(b)] as a function of the coordinate $\xi$, for all forcing amplitudes at $\Omega=18 \mathrm{rpm}$ and $\lambda_{f}=52.4 \mathrm{~cm}$. One can see that the wavelength and the normalized vorticity amplitude are nearly identical for the three lowest forcing amplitudes, indicating that the flow is in the linear regime. For larger values of the forcing amplitude $A$, the transverse (cross-beam) wavelength of the beam increases whereas the normalized beam vorticity decreases with $A$, indicating the emergence of nonlinear effects. Figure 13, showing a snapshot of the velocity field Fourier filtered at $\sigma_{0}$ for $A=$ $3.00 \mathrm{~mm}$ at $\Omega=18 \mathrm{rpm}$ and $\lambda_{f}=52.4 \mathrm{~cm}$, provides a direct illustration of the attractor thickening 


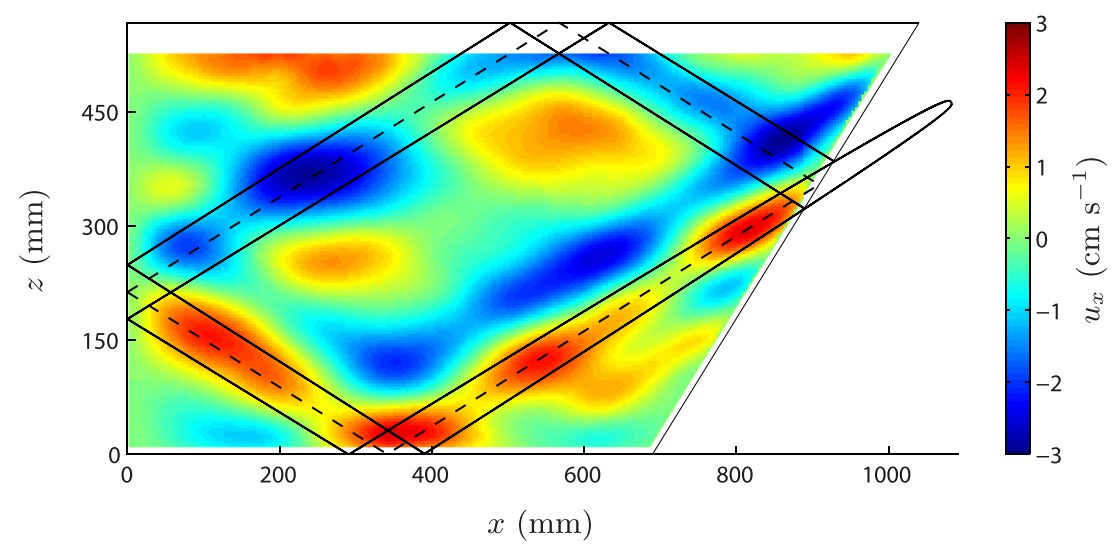

FIG. 13. Attractor in the nonlinear regime: snapshot of the velocity field in the vertical plane $y=y_{0}=L_{y} / 3$ Fourier filtered at the forcing frequency $\sigma_{0}$ for $A=3.00 \mathrm{~mm}, \lambda_{f}=52.4 \mathrm{~cm}$, and $\Omega=18 \mathrm{rpm}$. As in Fig. 8 , a sketch of the theoretical linear attractor is superimposed on the experimental field.

when increasing $A$. In this figure one still observes a concentration of energy around the theoretical attractor, but this concentration is clearly less pronounced than for low $A$ (see Fig. 8).

In Fig. 14 we report, as a function of the forcing Reynolds number $\operatorname{Re}_{f}=U_{f} \lambda_{f} / \nu$, the wavelength $\lambda$ [Fig. 14(a)] and the vorticity amplitude $W$ [Fig. 14(b)] averaged over the first branch of the attractor (the one following the focusing reflection). Three data series are reported here for $\Omega=3 \mathrm{rpm}$ and $\lambda_{f}=52.4 \mathrm{~cm}, \Omega=18 \mathrm{rpm}$ and $\lambda_{f}=52.4 \mathrm{~cm}$, and $\Omega=18 \mathrm{rpm}$ and $\lambda_{f}=26.2 \mathrm{~cm}$. In Fig. 14(a) the wavelength is normalized by $\ell^{2 / 3} L_{0}^{1 / 3}$ accounting for the dependence predicted by the linear attractor theory (9). We first note that the three data series collapse on a master curve. This suggests that the forcing Reynolds number $\mathrm{Re}_{f}$ is, to the first order, the parameter controlling the nonlinear evolution of the attractor. The normalized attractor wavelength $\lambda / \ell^{2 / 3} L_{0}^{1 / 3}$ is close to the value predicted by the linear theory $\Lambda \simeq 9.68$ for $\operatorname{Re}_{f} \lesssim 1000$ and increases at larger $\operatorname{Re}_{f}$ as already observed in Fig. 12(a) ( $\Lambda$ is the average of the theoretical prediction over the attractor first branch; it is shown by the horizontal dash-dotted line). One can however note that the normalized wavelength saturates at the larger Reynolds numbers for the series at $\Omega=3 \mathrm{rpm}$ and $\lambda_{f}=52.4 \mathrm{~cm}$ and at $\Omega=18 \mathrm{rpm}$ and $\lambda_{f}=26.2 \mathrm{~cm}$. This saturation is easy to understand: The wavelength $\lambda$ cannot be larger than the one of the forcing, since energy can only be transferred to smaller scales, via the focusing reflections. In Fig. 14(a) the horizontal solid lines (black, red, and blue) show the normalized wavelength $\lambda_{f} / \gamma$ for the three series of experiments. This wavelength theoretically corresponds to the wave excited by the wave maker after one reflection on the sloping wall. It stands as an upper limit for the wavelength found in the first branch of the attractor. For the largest forcing amplitudes at $\Omega=3 \mathrm{rpm}$ and $\lambda_{f}=52.4 \mathrm{~cm}$ and at $\Omega=18 \mathrm{rpm}$ and $\lambda_{f}=26.2 \mathrm{~cm}, \lambda$ approaches this limit, suggesting that almost no energy concentration in the attractor is observed. This is confirmed by the direct observation in Fig. 15 of two corresponding velocity fields at $\operatorname{Re}_{f} \simeq 9500$ in which one typically sees the wave excited by the wave maker reflecting on the sloping wall: Since the forcing wavelength is smaller than the theoretical wavelength expected for the nonlinear attractor, energy cannot be supplied to the latter by the forcing. In comparison, for the data series at $\Omega=18 \mathrm{rpm}$ and $\lambda_{f}=52.4 \mathrm{~cm}$, the wavelength for the largest Reynolds number $\operatorname{Re}_{f} \simeq 19000$ is still significantly lower than the excited wave original wavelength after one reflection $\lambda_{f} / \gamma$, revealing a greater robustness of the energy concentration in an attractor to the increase of Reynolds number when the rotation or the injection scale is larger, i.e., when the forcing Rossby number $\operatorname{Ro}_{f}=U_{f} / 2 \Omega \lambda_{f}$ is lower.

As mentioned in Sec. III, we expect that in an inviscid fluid the product of the velocity times the wavelength of an inertial wave is conserved during the reflection on a tilted wall. A tentative 

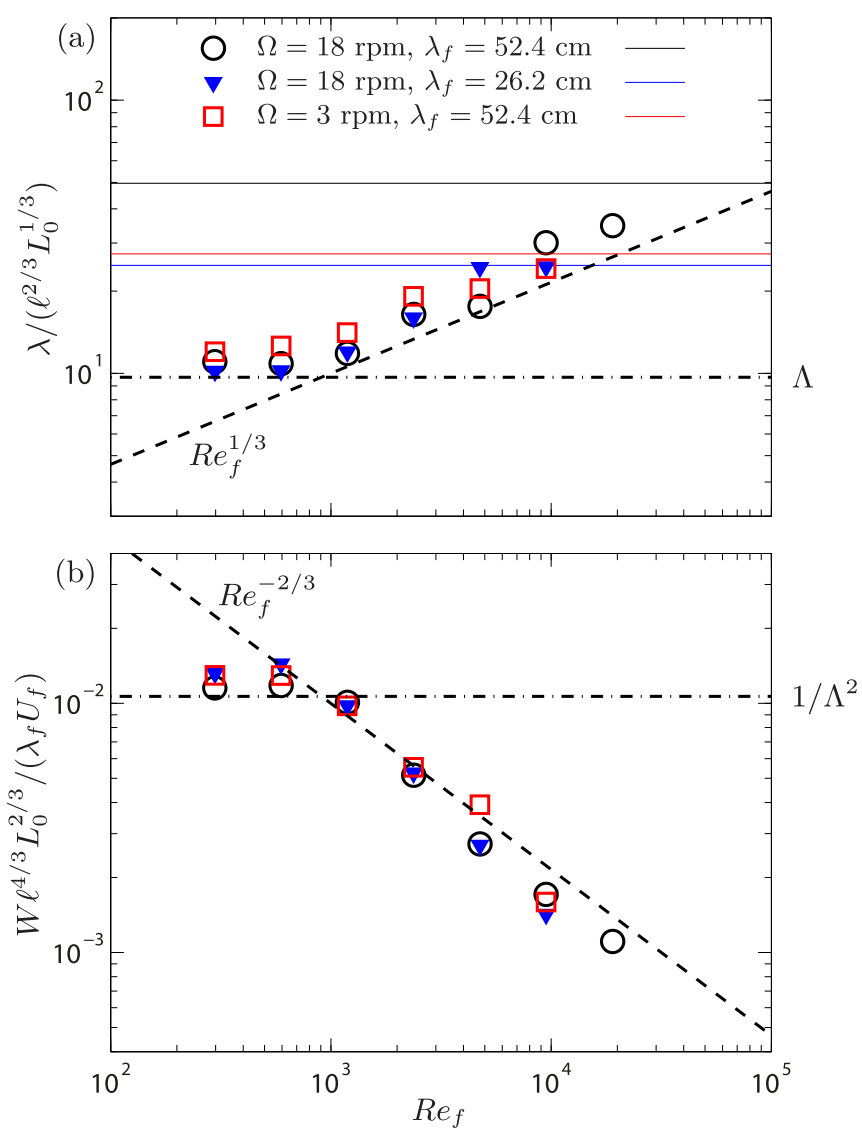

FIG. 14. (a) Normalized wavelength $\lambda / \ell^{2 / 3} L_{0}^{1 / 3}$ and (b) normalized vorticity amplitude $W \ell^{4 / 3} L_{0}^{2 / 3} / \lambda_{f} U_{f}$ averaged over the first branch of the attractor (the one following the focusing reflection) as a function of the forcing Reynolds number $\operatorname{Re}_{f}=U_{f} \lambda_{f} / \nu$. Squares correspond to experiments at $\Omega=3 \mathrm{rpm}$ and $\lambda_{f}=52.4 \mathrm{~cm}$, triangles to $\Omega=18 \mathrm{rpm}$ and $\lambda_{f}=26.2 \mathrm{~cm}$, and circles to $\Omega=18 \mathrm{rpm}$ and $\lambda_{f}=52.4 \mathrm{~cm}$. The dashed lines in (a) and in (b) show, respectively, the scaling laws $\lambda / \ell^{2 / 3} L_{0}^{1 / 3}=\operatorname{Re}_{f}^{1 / 3}$ and $W \ell^{4 / 3} L_{0}^{2 / 3} / \lambda_{f} U_{f}=\operatorname{Re}_{f}^{-2 / 3}$ predicted when replacing the fluid viscosity by the turbulent viscosity $v_{t}=U_{f} \lambda_{f}$ in the viscous length $\ell$ in the linear attractor model. In (a) the horizontal dash-dotted line shows the theoretical value $\Lambda$ for $\lambda / \ell^{2 / 3} L_{0}^{1 / 3}$ predicted by the linear attractor model described in Sec. III (average of the theoretical value over the first branch). In (b) the horizontal dash-dotted line shows the corresponding numerical value $1 / \Lambda^{2}$, which stands as an estimate for $W \ell^{4 / 3} L_{0}^{2 / 3} / \lambda_{f} U_{f}$ (see the main text). The solid horizontal lines, black, red, and blue, show the theoretical wavelength $\lambda_{f} / \gamma$ of the beam excited by the wave maker after one reflection on the sloping wall for the three experimental configurations, $\Omega=18 \mathrm{rpm}$ and $\lambda_{f}=52.4 \mathrm{~cm}, \Omega=3 \mathrm{rpm}$ and $\lambda_{f}=52.4 \mathrm{~cm}$, and $\Omega=18 \mathrm{rpm}$ and $\lambda_{f}=26.2 \mathrm{~cm}$, respectively.

scaling law for the vorticity amplitude of the linear attractor is therefore $W_{t}=U_{f} \lambda_{f} /\left(\ell^{2 / 3} L_{0}^{1 / 3}\right)^{2}$, where $U_{f}$ and $\lambda_{f}$ are characteristic of the wave initially forced by the wave maker and $\ell^{2 / 3} L_{0}^{1 / 3}$ is the theoretical scaling for the linear attractor wavelength. In Fig. 14(b) we report the vorticity amplitude $W$ normalized by $W_{t}$ as a function of $\operatorname{Re}_{f}$. This normalization collapses the three data series on a master curve which illustrates that $W_{t}$ catches the physics of the attractor amplitude in the linear and nonlinear regimes. We verify that this normalized vorticity $W / W_{t}$ is first constant at low forcing Reynolds number $\operatorname{Re}_{f} \lesssim 1000$, confirming the linear regime of the flow. A tentative estimate for the normalized attractor vorticity $W / W_{t}$ in the linear regime could be made by considering the 

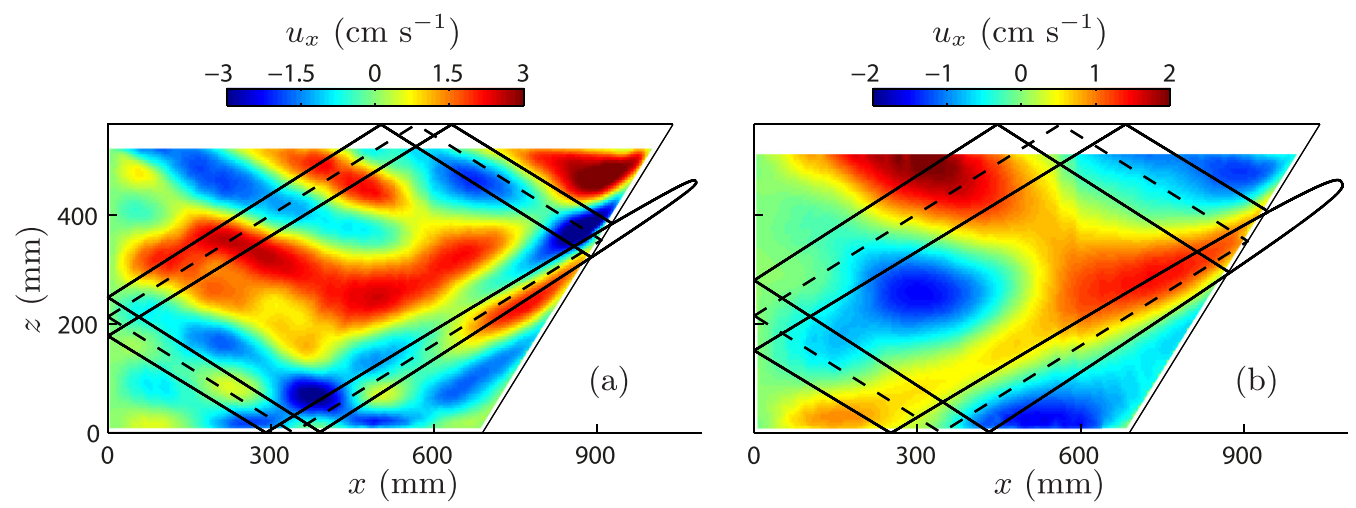

FIG. 15. Experiments without attractor: snapshots of two velocity fields in the vertical plane $y=y_{0}=L_{y} / 3$ at $\operatorname{Re}_{f}=9500$, Fourier filtered at $\sigma_{0}$ for (a) $A=6 \mathrm{~mm}, \lambda_{f}=26.2 \mathrm{~cm}$, and $\Omega=18 \mathrm{rpm}$ and (b) $A=18 \mathrm{~mm}$, $\lambda_{f}=52.4 \mathrm{~cm}$, and $\Omega=3 \mathrm{rpm}$. No attractor is observed in these fields because the wavelength of the theoretical nonlinear attractor is larger than the forcing wavelength. The absence of an attractor is therefore a combined effect of the nonlinearities and the forcing. As in Fig. 8, a sketch of the corresponding theoretical linear attractor is superimposed on each experimental field.

theoretical value predicted by the linear model for the attractor wavelength, i.e., $W / W_{t}=1 / \Lambda^{2} \simeq$ $1.07 \times 10^{-2}(\Lambda \simeq 9.68$ is the average over the first branch of the theoretical normalized attractor wavelength). This prediction for $W / W_{t}$ is reported with a horizontal dash-dotted line in Fig. 14(b): One sees that it indeed provides a reasonable estimate of the attractor vorticity in the linear regime. This behavior is consistent with the fact that the wavelength matches the linear theory in Fig. 14(a) for the same Reynolds number range. At larger $\operatorname{Re}_{f}$, the ratio $W / W_{t}$ decreases with $\operatorname{Re}_{f}$, revealing again the emergence of nonlinearities.

Figure 14 altogether allows us to state that the attractor wavelength $\lambda$ and vorticity amplitude $W$ follow the scaling laws predicted by the linear model but modified in the nonlinear regime by a prefactor function of the forcing Reynolds number

$$
\begin{gathered}
\lambda=\ell^{2 / 3} L_{0}^{1 / 3} f\left(\operatorname{Re}_{f}\right), \\
W=\frac{U_{f} \lambda_{f}}{\ell^{4 / 3} L_{0}^{2 / 3}} g\left(\operatorname{Re}_{f}\right) .
\end{gathered}
$$

When the wavelength $\lambda$ predicted by (14) is larger than $\lambda_{f} / \gamma$, no attractor can develop: A cutoff is therefore expected in (14) and (15) when $\operatorname{Re}_{f} \geqslant f^{-1}\left(\lambda_{f} / \gamma \ell^{2 / 3} L_{0}^{1 / 3}\right)$. As we will see in the following, the thickening of the attractor and the decrease of its relative amplitude when $\operatorname{Re}_{f}$ increases above $\operatorname{Re}_{f} \simeq 1000$ is correlated to the onset of a triadic resonance instability of the attractor. This instability drains energy from the mode at $\sigma_{0}$ toward lower-frequency modes. For the mode at $\sigma_{0}$, the instability can be seen as an additional dissipation to the viscous dissipation. A rudimentary but simple way to account for this additional dissipation is to replace the fluid viscosity by a turbulent viscosity $v_{t} \propto U_{f} \lambda_{f}$. Doing so in the viscous length $\ell \sim v^{1 / 2}$ appearing in Eqs. (14) and (15) leads to $f(\mathrm{Re})=\operatorname{Re}_{f}^{1 / 3}$ and $g(\mathrm{Re})=\operatorname{Re}_{f}^{-2 / 3}$. Reporting the laws (14) and (15) with these expressions in Figs. 14(a) and 14(b) provides an excellent description of the attractor wavelength and amplitude, confirming the relevance of the concept of turbulent viscosity to understand the nonlinear wave attractor. We note that no numerical prefactor has been used when reporting Eqs. (14) and (15) in Fig. 14.

In Fig. 16 we finally report the Reynolds number of the attractor defined as $\operatorname{Re}_{W}=W \lambda^{2} / v$ which is shown to increase linearly with the forcing Reynolds number $\operatorname{Re}_{f}$, over the whole range studied. The ratio $\mathrm{Re}_{W} / \mathrm{Re}_{f}$ indeed seems to be nearly constant for a given rotation rate: It is remarkably 


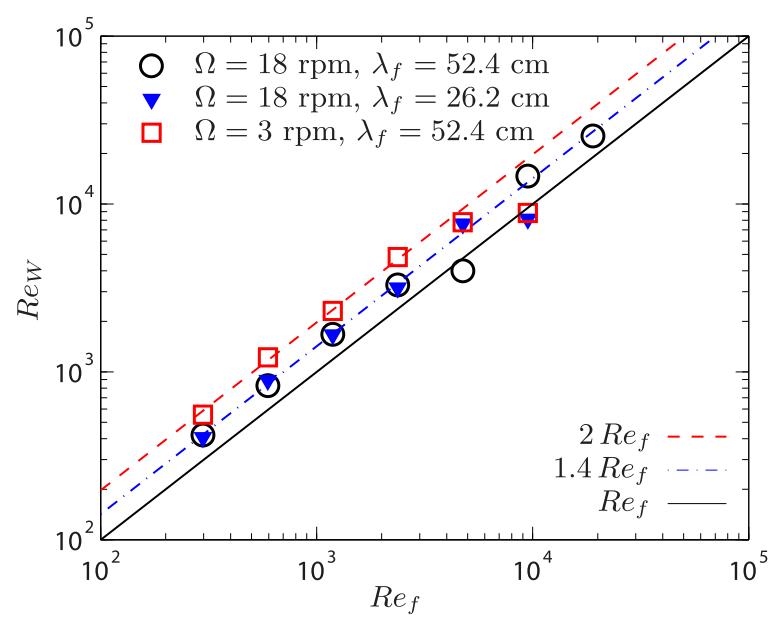

FIG. 16. Attractor Reynolds number $\mathrm{Re}_{W}=W \lambda^{2} / v$ averaged over the first branch of the attractor (the one following the focusing reflection) as a function of the forcing Reynolds number $\operatorname{Re}_{f}=U_{f} \lambda_{f} / \nu$. Squares correspond to experiments at $\Omega=3 \mathrm{rpm}$ and $\lambda_{f}=52.4 \mathrm{~cm}$, triangles to $\Omega=18 \mathrm{rpm}$ and $\lambda_{f}=26.4 \mathrm{~cm}$, and circles to $\Omega=18 \mathrm{rpm}$ and $\lambda_{f}=52.4 \mathrm{~cm}$.

almost unaffected by the onset of the attractor instability at $\operatorname{Re}_{f} \simeq 1000$. The ratio $\operatorname{Re}_{W} / \operatorname{Re}_{f}$ is nevertheless slowly dependent on $\Omega$ with $\operatorname{Re}_{W} / \operatorname{Re}_{f} \simeq 2.0$ for $\Omega=3 \mathrm{rpm}$ and $\operatorname{Re}_{W} / \operatorname{Re}_{f} \simeq 1.4$ for $\Omega=18 \mathrm{rpm}$. Since one would expect $\operatorname{Re}_{W} / \operatorname{Re}_{f} \simeq 1$ if a simple and single reflection of the forced wave is observed, the ratio $\operatorname{Re}_{W} / \operatorname{Re}_{f}$ can be seen as a quantifier of the presence of an attractor. Following (14) and (15), one has $\operatorname{Re}_{W} / \operatorname{Re}_{f}=g\left(\operatorname{Re}_{f}\right) f\left(\operatorname{Re}_{f}\right)^{2}$. The weak but clear dependence of $\operatorname{Re}_{W} / \operatorname{Re}_{f}$ with the rotation rate $\Omega$ that we report here shows that $f=\lambda / \ell^{2 / 3} L_{0}^{1 / 3}$ and $g=W \ell^{4 / 3} L_{0}^{2 / 3} / \lambda_{f} U_{f}$ are weakly dependent on the cavity Ekman number Ek $=v / 2 \Omega H^{2}$ in addition to the leading dependence on the Reynolds number $\operatorname{Re}_{f}$. Since this weak dependence does not involve the forcing wavelength $\lambda_{f}$ and amplitude $U_{f}$, it might be related to the physics of Ekman viscous boundary layers on the walls of the cavity. We are however currently not able to propose an explanation for this behavior, which is weak but significant.

\section{Triadic resonance instability}

To further understand the nonlinear evolution of the flow beyond the instability threshold of the attractor, we report in Fig. 17 the temporal energy spectrum $E(\sigma)$ [Eq. (12)] for all experiments at $\Omega=18 \mathrm{rpm}$ and $\lambda_{f}=52.4 \mathrm{~cm}$. Beyond $A=0.38 \mathrm{~mm}\left(\operatorname{Re}_{f} \simeq 1200\right)$, at which the linear prediction for the attractor thickness and amplitude start to fail, we observe the emergence of two subharmonic bumps in the spectrum. The frequencies $\sigma_{1}$ and $\sigma_{2}$ around which the bumps are centered are consistent with a triadic resonance with the forcing frequency $\sigma_{0}$, i.e., $\sigma_{1}+\sigma_{2}=\sigma_{0}$, as can be seen in Table I. We recall that the energetic peak at frequency $\sigma^{*}=0.5$, i.e., $\sigma=\Omega$, observed for the lower amplitudes $A$, corresponds to the tilt-over flow forced by the earth's rotation which induces a Coriolis force on the fluid moving in the laboratory [13,47]. This peak probably hides the expected second energy bump in the experiments at $A=0.38 \mathrm{~mm}$ for which we report only one subharmonic frequency $\sigma_{1}$. We also highlight that the sharp peak observed at $\sigma^{*}=0.35$, i.e., $\sigma=\sigma_{0}-\Omega$, for the low-forcing experiments corresponds to the interaction of this tilt-over flow with the forcing frequency $\sigma_{0}$.

In any case, we can highlight that the emergence of the subharmonic instability through a triadic resonance illustrated by Fig. 17 is fully correlated with the increase of the attractor length scale and to the damping of its normalized amplitude revealed in Figs. 12 and 14. The subharmonic bumps in 


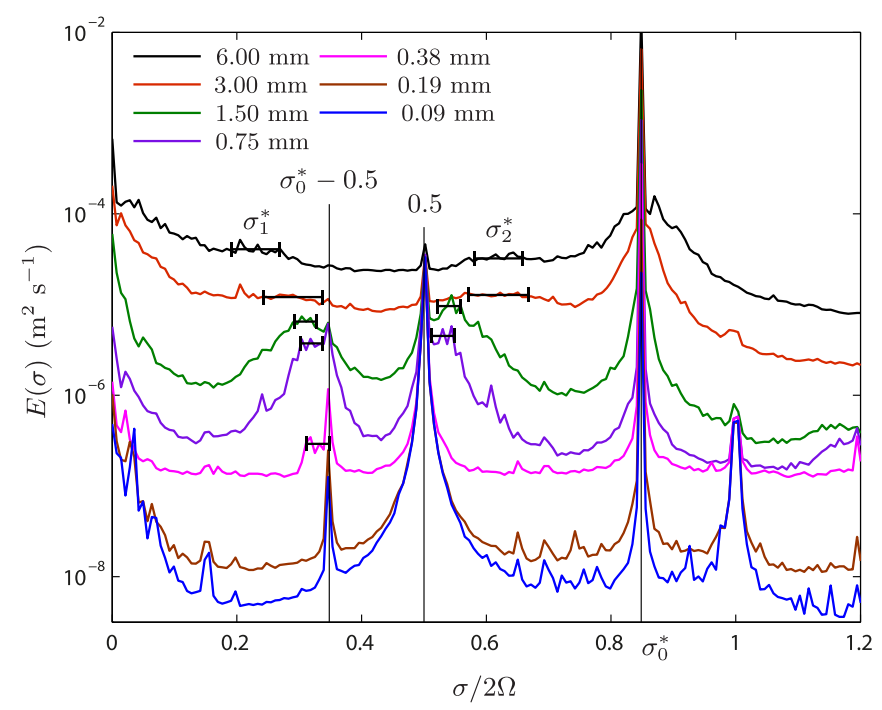

FIG. 17. Temporal power spectral density $E(\sigma)$ [Eq. (12)] as a function of the normalized frequency $\sigma^{*}=\sigma / 2 \Omega$ for all forcing amplitudes $A$ at $\Omega=18 \mathrm{rpm}$ and $\lambda_{f}=52.4 \mathrm{~cm}$. For each spectrum, the horizontal error bars indicate the frequency intervals around which the subharmonic bumps are centered. These intervals correspond to the frequencies reported in Table I. We have highlighted with vertical lines three other energy peaks at frequencies $\sigma_{0}^{*}=\sigma_{0} / 2 \Omega, \sigma^{*}=0.5$ (i.e., $\sigma=\Omega$ ), and $\sigma^{*}=\sigma_{0}^{*}-0.5$.

the temporal spectra are wide, a feature that was already reported for the triadic resonance instability of an experimental plane inertial wave in [8]. It confirms that there is a specificity for experimental inertial waves with respect to internal waves [37,44] and numerical inertial waves [36] for which triadic instability produces two precise frequencies.

In Fig. 17, for the two largest forcing amplitudes $A=3 \mathrm{~mm}$ and $6 \mathrm{~mm}\left(\operatorname{Re}_{f} \simeq 9500\right.$ and 19000), the subharmonic bumps become hardly distinguishable. We believe that this last feature does not mean that the instability has vanished since the total energy stored in the modes at subharmonic frequencies is still significant but spread over large frequency ranges. The horizontal error bars shown in Fig. 17 aim at representing qualitatively the uncertainty on the determination of the central frequency of the subharmonic bumps $\sigma_{1}^{*}$ and $\sigma_{2}^{*}$. Thus, considering with precaution the spectra for the two largest amplitudes in Fig. 17, we can note that the separation between the bumps' center frequencies $\sigma_{1}^{*}$ and $\sigma_{2}^{*}$ seems to increase with $A, \sigma_{1}^{*}$ and $\sigma_{2}^{*}$ going farther away from $\sigma_{0}^{*} / 2$. The bumps seem at the same time to get wider, whereas their amplitudes $E\left(\sigma_{1,2}\right)$ progressively decrease

TABLE I. Normalized center frequencies $\sigma_{1}^{*}=\sigma_{1} / 2 \Omega$ and $\sigma_{2}^{*}=\sigma_{2} / 2 \Omega$ of the subharmonic bumps observed in the temporal energy spectra for $\Omega=18 \mathrm{rpm}$ and $\lambda_{f}=52.4 \mathrm{~cm}$ (Fig. 17) as a function of the forcing amplitude $A$. Cells with no entry correspond to cases when no bump is observable.

\begin{tabular}{lrccc}
\hline \hline$A(\mathrm{~mm})$ & $\operatorname{Re}_{f}$ & $\sigma_{1}^{*}$ & $\sigma_{2}^{*}$ & $\left(\sigma_{1}+\sigma_{2}\right) / \sigma_{0}$ \\
\hline 0.09 & 300 & & & \\
0.19 & 600 & & & \\
0.38 & 1190 & $0.32 \pm 0.01$ & & \\
0.75 & 2380 & $0.32 \pm 0.02$ & $0.53 \pm 0.02$ & $1.00 \pm 0.05$ \\
1.50 & 4750 & $0.31 \pm 0.02$ & $0.54 \pm 0.02$ & $1.00 \pm 0.05$ \\
3.00 & 9500 & $0.29 \pm 0.05$ & $0.62 \pm 0.05$ & $1.07 \pm 0.12$ \\
6.00 & 19000 & $0.23 \pm 0.04$ & $0.64 \pm 0.04$ & $1.02 \pm 0.09$ \\
\hline \hline
\end{tabular}


(a) $A=0.75 \mathrm{~mm}, \sigma^{*}=0.32$

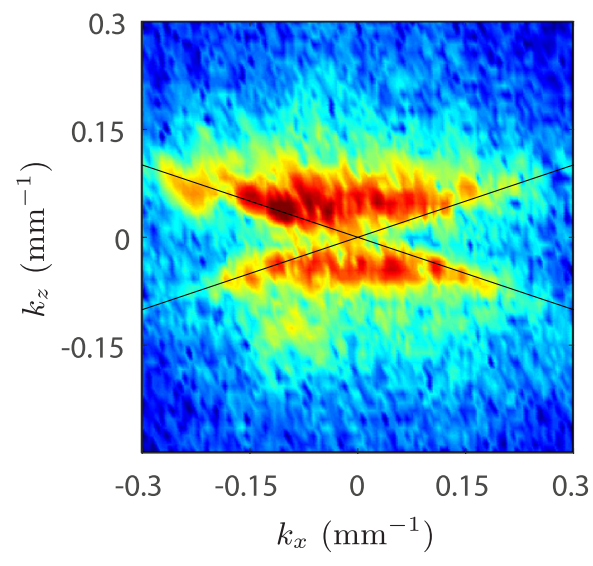

(c) $A=3.00 \mathrm{~mm}, \sigma^{*}=0.29$

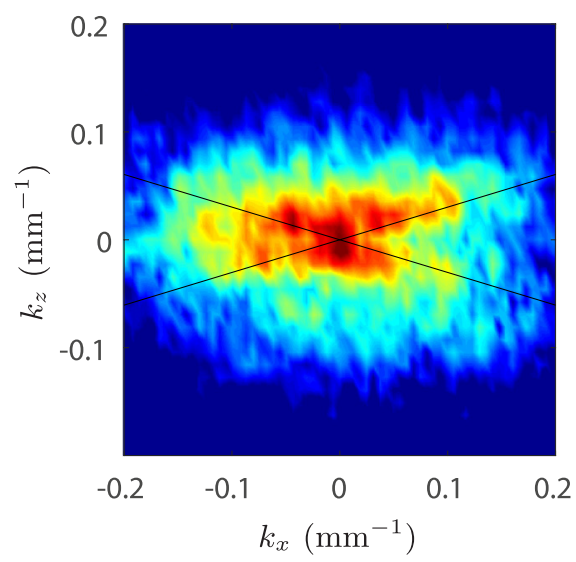

(b) $A=0.75 \mathrm{~mm}, \sigma^{*}=0.53$

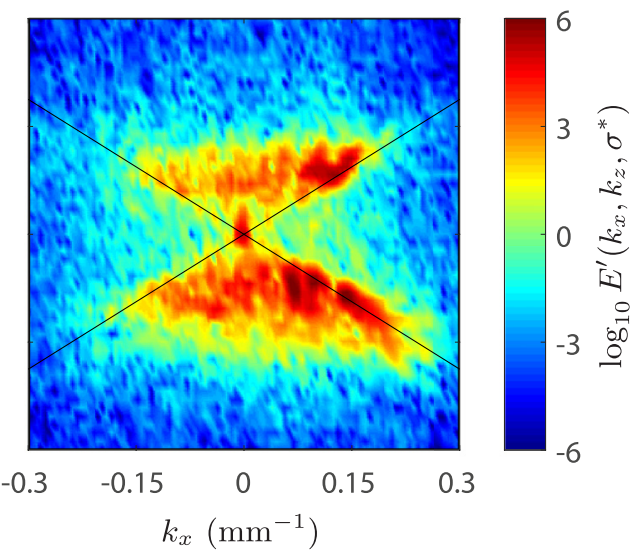

(d) $A=3.00 \mathrm{~mm}, \sigma^{*}=0.62$

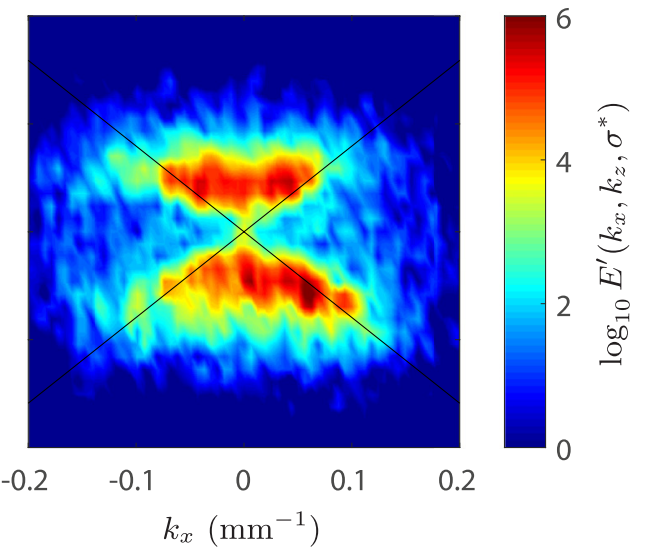

FIG. 18. Spatiotemporal power spectral density $E^{\prime}\left(k_{x}, k_{z}, \sigma\right)$ [Eq. (16)] for the experiments at $\Omega=18 \mathrm{rpm}$, $\lambda_{f}=52.4 \mathrm{~cm}$, and (a) and (b) $A=0.75 \mathrm{~mm}\left(\operatorname{Re}_{f} \simeq 2400\right)$ and (c) and (d) $A=3.00 \mathrm{~mm}\left(\operatorname{Re}_{f} \simeq 9500\right)$. The selected frequencies for each experiment correspond to the center frequencies of the subharmonic bumps in their temporal spectrum, i.e., (a) $\sigma^{*}=0.32$ and (b) $\sigma^{*}=0.53$ for $A=0.75 \mathrm{~mm}$ and (c) $\sigma^{*}=0.29$ and (d) $\sigma^{*}=0.62$ for $A=3.00 \mathrm{~mm}$ (see Table I). In each panel, black lines correspond to the dispersion relation $\left|\sigma^{*}\right|=\left|k_{z}\right| /\left(k_{x}^{2}+k_{z}^{2}\right)^{1 / 2}$ of inertial waves with $k_{y}=0$.

with $A$ relative to the base level of the spectrum. These results are in discrepancy with the temporal spectra reported in [36] for numerical simulations of inertial wave attractor in which $\sigma_{1}^{*}$ and $\sigma_{2}^{*}$ are clearly defined by sharp peaks and tend toward $\sigma_{0}^{*} / 2$ as the forcing amplitude increases. These points remain to be understood and will be discussed in Sec. V.

To confirm that the flow components associated with the bumps at $\sigma_{1}$ and $\sigma_{2}$ are composed of inertial waves, we search in the following for the spatiotemporal signature of their dispersion relation. To do so, we compute for each experiment the normalized spatiotemporal power spectral density of the velocity field as

$$
E^{\prime}\left(k_{x}, k_{z}, \sigma\right)=\frac{\left|\tilde{u}_{j}^{\prime}\left(k_{x}, k_{z}, \sigma\right)\right|^{2}}{\left\langle\left|\tilde{u}_{j}^{\prime}\left(k_{x}, k_{z}, \sigma\right)\right|^{2}\right\rangle},
$$



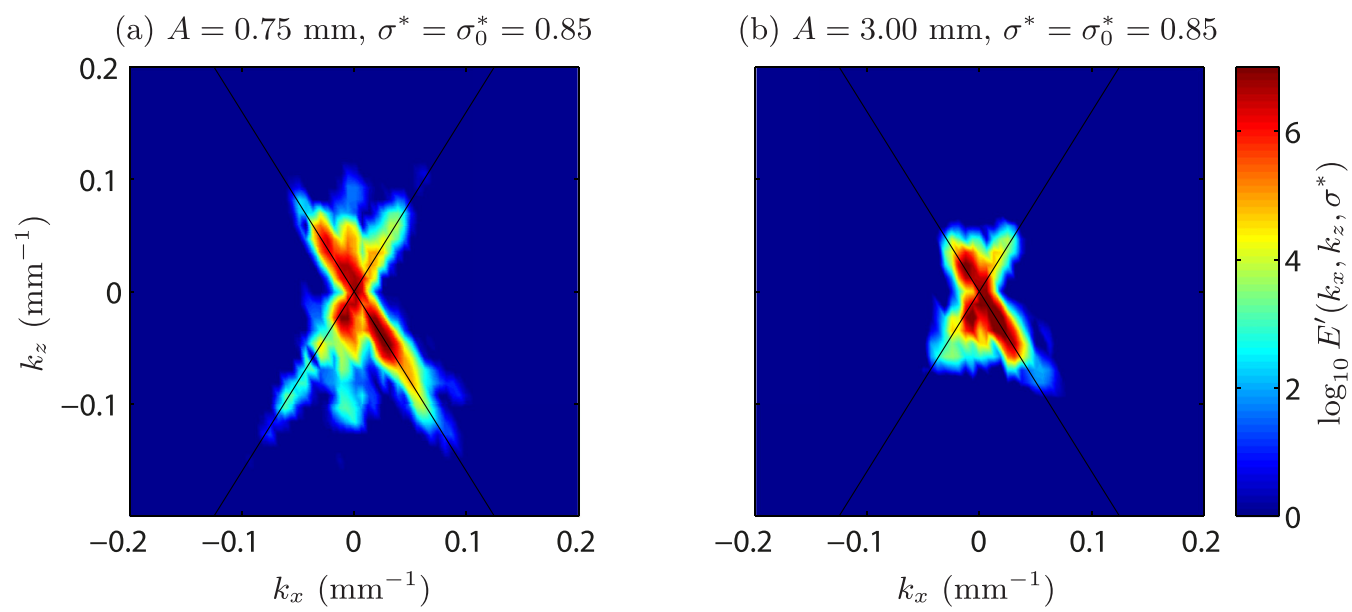

FIG. 19. Spatiotemporal power spectral density $E^{\prime}\left(k_{x}, k_{z}, \sigma\right)$ [Eq. (16)] at the forcing frequency $\sigma=\sigma_{0}$ for the experiments at $\Omega=18 \mathrm{rpm}, \lambda_{f}=52.4 \mathrm{~cm}$, and (a) $A=0.75 \mathrm{~mm}\left(\operatorname{Re}_{f} \simeq 2400\right)$ and (b) $A=3.00 \mathrm{~mm}$ $\left(\operatorname{Re}_{f} \simeq 9500\right)$. In each panel, black lines correspond to the dispersion relation $\left|\sigma^{*}\right|=\left|k_{z}\right| /\left(k_{x}^{2}+k_{z}^{2}\right)^{1 / 2}$ of inertial waves with $k_{y}=0$.

where

$$
\tilde{u}_{j}^{\prime}\left(k_{x}, k_{z}, \sigma\right)=\frac{1}{(2 \pi)^{3}} \int_{0}^{T} \int_{0}^{L_{x}} \int_{0}^{H} u_{j}\left(x, y_{0}, z, t\right) e^{-i\left(\sigma t+k_{x} x+i k_{z} z\right)} d t d x d z
$$

is the spatiotemporal Fourier transform of $u_{j}\left(x, y_{0}, z, t\right)$ with $j=x, z$ and the angular brackets represent the average over wave-number space (normalization by the energy at $\sigma$ ).

In Fig. 18 we report this spatiotemporal spectrum $E^{\prime}\left(k_{x}, k_{z}, \sigma\right)$ for the experiments at $\Omega=$ $18 \mathrm{rpm}, \lambda_{f}=52.4 \mathrm{~cm}$, and $A=0.75 \mathrm{~mm}\left(\operatorname{Re}_{f} \simeq 2400\right)$ or $A=3.00 \mathrm{~mm}\left(\operatorname{Re}_{f} \simeq 9500\right)$, for the respective center frequencies of the bumps in their temporal spectrum. Black lines represent the dispersion relation $\left|\sigma^{*}\right|=\left|k_{z}\right| /\left(k_{x}^{2}+k_{z}^{2}\right)^{1 / 2}$ of $2 \mathrm{D}$ inertial waves invariant in the $y$ direction, i.e., with $k_{y}=0$. In such a representation, inertial waves with their wave vector in the measurement plane will appear through energy concentration on the black lines.

In Fig. 18 the energetic regions of the spatiotemporal spectra resemble a sandglass with several maxima of energy along the black lines. This shows that the flow component at frequencies $\sigma_{1}$ and $\sigma_{2}$ is composed of inertial waves of which a significant proportion has a nonzero wave-vector component in the $y$ direction. Indeed, the general expression of the dispersion relation is $\left|\sigma^{*}\right|=$ $\left|k_{z}\right| /\left(k_{x}^{2}+k_{y}^{2}+k_{z}^{2}\right)^{1 / 2}$, and inertial waves with $k_{y} \neq 0$ will show up with energy in the regions between the two black lines $\left[\left|\sigma^{*}\right|=\left|k_{z}\right| /\left(k_{x}^{2}+k_{z}^{2}\right)^{1 / 2}\right]$ and containing the axis $k_{x}=0$. Considering other forcing amplitude $A$ and other frequencies $\sigma^{*}$ inside the subharmonic bumps of the temporal spectra leads to similar spatiotemporal spectra. The only exception is the spatiotemporal spectra at the forcing frequency $\sigma_{0}$ in which the energy is clearly concentrated on the black lines, as can be seen in Fig. 19 (same experiments as in Fig. 18). This result confirms that the attractor remains nearly two dimensional, with $k_{y} \simeq 0$, in the measurement plane $y=L_{y} / 3$. For other frequencies $\sigma^{*}$, the presence of waves with wave vector in the measurement plane $y=y_{0}$ is natural since the triadic instability of an attractor invariant in the $y$ direction leads to such waves if no spontaneous breaking of symmetry appears. Waves found here with $k_{y} \neq 0$ might be the fruit of the nonperfect $y$ invariance of the experimental attractor due to the finite size in the $y$ direction of the wave maker and/or to the presence of viscous boundary layers on the vertical walls of the cavity at $y=0$ and $y=L_{y}$, which both should lead to some three dimensionality of the flow. This three dimensionality might also be at the origin of the previously highlighted differences (bumps spreading in frequency 
and moving away from each other with increasing $A$ ) of the temporal spectra with the simulations of Jouve and Ogilvie [36], which were strictly two dimensional, invariant in the $y$ direction.

\section{CONCLUSION}

In this article we have reported PIV measurements of the flow generated by a large-scale harmonic inertial forcing in a trapezoidal cavity with a tilted wall submitted to a global rotation. In the linear regime, we observed a concentration of the energy along a limit cycle inside the cavity as expected from the theory of internal wave attractors. Our data showed that the model, initially proposed by Rieutord et al. [28] followed by Grisouard et al. [35] and Jouve and Ogilvie [36], describing attractors as a portion of a self-similar wave beam emitted by a virtual point source upstream of the tilted wall accounts correctly for the measured values of the wavelength in the attractor as well as for the scaling laws of the spatial decay of its amplitude.

We have further explored the nonlinear regime of the attractor. The following scenario is observed when increasing the forcing amplitude. The attractor becomes unstable beyond a forcing Reynolds number of $\operatorname{Re}_{f} \simeq 1200$. This instability feeds inertial waves gathered around two subharmonic frequencies $\sigma_{1}$ and $\sigma_{2}$ resonant with the attractor frequency $\sigma_{0}$. This triadic resonance instability is accompanied by a thickening in size and a damping in relative amplitude of the attractor as the forcing amplitude grows above the instability threshold. In parallel, the two bumps corresponding to the subharmonic waves in the temporal spectrum have their central frequencies $\sigma_{1}$ and $\sigma_{2}$ gradually moving away from $\sigma_{0} / 2$ while the bumps spread over wider ranges of frequencies tending to build a continuum of energy in frequency.

In [38], from similar experiments with internal gravity waves, Brouzet et al. also report an increase of the attractor wavelength and a reduction of its relative amplitude when the attractor becomes unstable via a triadic resonance. They interpreted their results by introducing a turbulent viscosity accounting for the fact the instability creates a sink of energy for the attractor. In this article, by considering data for two different rotation rates $\Omega$ and for two forcing wavelengths $\lambda_{f}$, we have demonstrated that the attractor mean wavelength $\lambda$ and vorticity amplitude $W$ follow scaling laws predicted by the linear attractor model even in the nonlinear regime if one uses the turbulent viscosity $v_{t}=U_{f} \lambda_{f}$ based on the forcing velocity $U_{f}$ and wavelength $\lambda_{f}$ in place of the fluid kinetic viscosity. This framework eventually predicts that the attractor wavelength $\lambda$ and vorticity $W$ follow power laws $\lambda \sim \operatorname{Re}_{f}^{1 / 3}$ and $W \sim \operatorname{Re}_{f}^{-2 / 3}$ with the forcing Reynolds number $\operatorname{Re}_{f}$ beyond the onset of the triadic instability whose scalings are in clear agreement with our data.

Regarding the subharmonic waves produced by the instability, it is worth highlighting two major differences from previous results $[36-38,44]$. The first one relies on the fact that the instability provides energy to large ranges of frequencies and not to two precise subharmonic frequencies. This weak selectivity is not specific to attractors since it has also been reported for a plane inertial wave beam [8]. It is in clear discrepancy with the strong selectivity reported for numerical inertial waves [36]. It might be related to the three-dimensional nature of the velocity oscillations in inertial waves: In a $y$ invariant $\left(k_{y}=0\right)$ wave at frequency $\sigma$, fluid particles describe circular translations in planes tilted by an angle $\theta=\cos ^{-1}(\sigma / 2 \Omega)$ and velocity oscillations are observed in the three directions $x, y$, and $z$. The vertical walls of the cavity are therefore incompatible with such $y$ invariance in inertial wave experiments. This feature is on the contrary absent in 2D numerical simulations of inertial waves in which periodic boundary conditions are used in the $y$ direction [36]. In the case of internal gravity waves [37,38,44], for which fluid particles oscillate only in the vertical plane $(x, z)$, the physical vertical walls $y=0$ and $y=L_{y}$ are compatible with inviscid boundary conditions. The three-component character of inertial waves interacting with physical walls therefore appears as a good candidate to explain the weak selectivity of the triadic instability. This could be tested by comparing the results of numerical simulations with $y$ periodic boundary conditions and with walls at $y=0$ and $y=L_{y}$. In this context, it is worth noting that Manders and Maas [31] have studied the three-dimensional structure of an experimental inertial wave attractor forced by a libration perturbation of the global rotation in a trapezoidal cavity. The dependence 
of the experimental attractor in the $y$ direction is however probably strongly different compared to the one in the data we report here since the libration forcing is antisymmetric for mirror reflection with respect to the plane $y=L / 2$, leading to a phase shift of $\pi$ in the attractor between half space $y<L / 2$ and half space $y>L / 2$. The libration forcing actually induces an oscillating horizontal circulation in the plane $(x, y)$ with strong horizontal velocities close to the vertical walls, which is also a feature absent in our experiments.

A last major difference from previous works is that the instability of the inertial wave attractor reported here leads to subharmonic waves with frequencies more and more remote from $\sigma_{0} / 2$ as the forcing amplitude grows. This behavior is the opposite of the one reported in numerical simulations of an inertial wave attractor in a tilted square [36]. It is also in apparent contradiction with the theory of triadic instability of plane inertial or internal waves $[8,55]$, which predicts that the frequencies of the two subharmonic waves tend toward $\sigma_{0} / 2$ as the Reynolds number of the primary wave increases. This behavior is probably the most intriguing that has been reported here.

\section{ACKNOWLEDGMENTS}

We acknowledge M. Rabaud and F. Moisy for fruitful discussions and J. Amarni, A. Aubertin, L. Auffray, and R. Pidoux for experimental help. This work was supported by the Agence Nationale de la Recherche through Grant "DisET” No. ANR-17-CE30-0003.

[1] H. Greenspan, The Theory of Rotating Fluids (Cambridge University Press, Cambridge, 1968).

[2] J. Pedlosky, Geophysical Fluid Dynamics (Springer, New York, 1987).

[3] D. E. Mowbray and B. S. H. Rarity, A theoretical and experimental investigation of the phase configuration of internal waves of small amplitude in a density stratified liquid, J. Fluid Mech. 28, 1 (1967).

[4] M. R. Flynn, K. Onu, and B. R. Sutherland, Internal wave excitation by a vertically oscillating sphere, J. Fluid Mech. 494, 65 (2003).

[5] P.-P. Cortet, C. Lamriben, and F. Moisy, Viscous spreading of an inertial wave beam in a rotating fluid, Phys. Fluids 22, 086603 (2010).

[6] N. Machicoane, P.-P. Cortet, B. Voisin, and F. Moisy, Influence of the multipole order of the source on the decay of an inertial wave beam in a rotating fluid, Phys. Fluids 27, 066602 (2015).

[7] M. Mercier, D. Martinand, M. Mathur, L. Gostiaux, T. Peacock, and T. Dauxois, New wave generation, J. Fluid Mech. 657, 308 (2010).

[8] G. Bordes, F. Moisy, T. Dauxois, and P.-P. Cortet, Experimental evidence of a triadic resonance of plane inertial waves in a rotating fluid, Phys. Fluids 24, 014105 (2012).

[9] K. D. Aldridge and A. Toomre, Axisymmetric inertial oscillations of a fluid in a rotating spherical container, J. Fluid Mech. 37, 307 (1969).

[10] A. D. McEwan, Inertial oscillations in a rotating fluid cylinder, J. Fluid Mech. 40, 603 (1970).

[11] L. R. M. Maas, On the amphidromic structure of inertial waves in rectangular parallelepiped, Fluid Dyn. Res. 33, 373 (2003).

[12] J. Boisson, C. Lamriben, L. R. M. Maas, P.-P. Cortet, and F. Moisy, Inertial waves and modes excited by the libration of a rotating cube, Phys. Fluids 24, 076602 (2012).

[13] J. Boisson, D. Cébron, F. Moisy, and P.-P. Cortet, Earth rotation prevents exact solid-body rotation of fluids in the laboratory, Europhys. Lett. 98, 59002 (2012).

[14] J. Lighthill, Waves in Fluids (Cambridge University Press, Cambridge, 1978).

[15] K. K. Zhang, X. H. Liao, and P. Earnshaw, On inertial waves and oscillations in a rapidly rotating spheroid, J. Fluid Mech. 504, 1 (2004).

[16] M. Rieutord, Linear theory of rotating fluids using spherical harmonics part II, time-periodic flows, Geophys. Astrophys. Fluid Dyn. 59, 185 (1991). 
[17] A. Tilgner, Driven inertial oscillations in spherical shells, Phys. Rev. E 59, 1789 (1999).

[18] J. Noir, F. Hemmerlin, J. Wicht, S. M. Baca, and J. M. Aurnou, An experimental and numerical study of librationally driven flow in planetary cores and subsurface oceans, Phys. Earth Planet. Inter. 173, 141 (2009).

[19] M. A. Calkins, J. Noir, J. D. Eldredge, and J. M. Aurnou, Axisymmetric simulations of libration-driven fluid dynamics in a spherical shell geometry, Phys. Fluids 22, 086602 (2010).

[20] F. H. Busse, Steady fluid flow in a precessing spheroidal shell, J. Fluid Mech. 33, 739 (1968).

[21] R. R. Kerswell, On the internal shear layers spawned by the critical regions in oscillatory Ekman boundary layers, J. Fluid Mech. 298, 311 (1995).

[22] J. Noir, D. Jault, and P. Cardin, Numerical study of the motions within a slowly precessing sphere at low Ekman number, J. Fluid Mech. 437, 283 (2001).

[23] S. T. Suess, Viscous flow in a deformable rotating container, J. Fluid Mech. 45, 189 (1971).

[24] C. Morize, M. Le Bars, P. Le Gal, and A. Tilgner, Experimental Determination of Zonal Winds Driven by Tides, Phys. Rev. Lett. 104, 214501 (2010).

[25] O. M. Phillips, Energy transfer in rotating fluids by reflection of inertial waves, Phys. Fluids 6, 513 (1963).

[26] L. R. M. Maas and F.-P. A. Lam, Geometric focusing of internal waves, J. Fluid Mech. 300, 1 (1995).

[27] L. R. M. Maas, D. Benielli, J. Sommeria, and F.-P. A. Lam, Observation of an internal wave attractor in a confined, stably stratified fluid, Nature (London) 388, 557 (1997).

[28] M. Rieutord, B. Georgeot, and L. Valdettaro, Inertial waves in a rotating spherical shell: Attractors and asymptotic spectrum, J. Fluid Mech. 435, 103 (2001).

[29] A. M. M. Manders and L. R. M. Maas, Observations of inertial waves in a rectangular basin with one sloping boundary, J. Fluid Mech. 493, 59 (2003).

[30] L. R. M. Maas, Wave focusing and ensuing mean flow due to symmetry breaking in rotating fluids, J. Fluid Mech. 437, 13 (2001).

[31] A. M. M. Manders and L. R. M. Maas, On the three-dimensional structure of the inertial wave field in a rectangular basin with one sloping boundary, Fluid Dyn. Res. 35, 1 (2004).

[32] M. Klein, T. Seelig, M. V. Kurgansky, A. Ghasemi, I. D. Borcia, A. Will, E. Schaller, C. Egbers, and U. Harlander, Inertial wave excitation and focusing in a liquid bounded by a frustum and a cylinder, J. Fluid Mech. 751, 255 (2014).

[33] G. I. Ogilvie, Wave attractors and the asymptotic dissipation rate of tidal disturbances, J. Fluid Mech. 543, 19 (2005).

[34] J. Hazewinkel, P. van Breevoort, S. Dalziel, and L. R. M. Maas, Observations on the wavenumber spectrum and evolution of an internal wave attractor, J. Fluid Mech. 598, 373 (2008).

[35] N. Grisouard, C. Staquet, and I. Pairaud, Numerical simulation of a two-dimensional internal wave attractor, J. Fluid Mech. 614, 1 (2008).

[36] L. Jouve and G. I. Ogilvie, Direct numerical simulations of an inertial wave attractor in linear and nonlinear regimes, J. Fluid Mech. 745, 223 (2014).

[37] H. Scolan, E. Ermanyuk, and T. Dauxois, Nonlinear Fate of Internal Waves Attractors, Phys. Rev. Lett. 110, 234501 (2013).

[38] C. Brouzet, I. N. Sibgatullin, E. V. Ermanyuk, S. Joubaud, and T. Dauxois, Scale effects in internal wave attractors, Phys. Rev. Fluids 2, 114803 (2017).

[39] N. Machicoane, V. Labarre, B. Voisin, F. Moisy, and P.-P. Cortet, Wake of inertial waves of a horizontal cylinder in horizontal translation, Phys. Rev. Fluids 3, 034801 (2018).

[40] F. Beckebanze, C. Brouzet, I. N. Sibgatullin, and L. R. M. Maas, Damping of quasi-two-dimensional internal wave attractors by rigid-wall friction, J. Fluid Mech. 841, 614 (2018).

[41] C. Brouzet, E. Ermanyuk, S. Joubaud, G. Pillet, and T. Dauxois, Internal wave attractors: Different scenarios of instability, J. Fluid Mech. 811, 544 (2017).

[42] D. W. Moore and P. G. Saffman, The structure of free vertical shear layers in a rotating fluid and the motion produced by a slowly rising body, Philos. Trans. R. Soc. London Ser. A 264, 597 (1969).

[43] N. H. Thomas and T. N. Stevenson, A similarity solution for viscous internal waves, J. Fluid Mech. 54, 495 (1972). 
[44] B. Bourget, T. Dauxois, S. Joubaud, and P. Odier, Experimental study of parametric subharmonic instability for internal waves, J. Fluid Mech. 723, 1 (2013).

[45] B. Bourget, H. Scolan, T. Dauxois, M. Le Bars, P. Odier, and S. Joubaud, Finite-size effects in parametric subharmonic instability, J. Fluid Mech. 759, 739 (2014).

[46] H. H. Karimi and T. R. Akylas, Parametric subharmonic instability of internal waves: Locally confined beams versus monochromatic wave trains, J. Fluid Mech. 757, 381 (2014).

[47] S. A. Triana, D. S. Zimmerman, and D. P. Lathrop, Precessional states in a laboratory model of the earth's core, J. Geophys. Res. 117, B04103 (2012).

[48] S. Kida, Steady flow in a rapidly rotating sphere with weak precession, J. Fluid Mech. 680, 150 (2011).

[49] S. Sakai, The horizontal scale of rotating convection in the geostrophic regime, J. Fluid Mech. 333, 85 (1997).

[50] E. M. King and J. M. Aurnou, Thermal evidence for Taylor columns in turbulent rotating Rayleigh-Bénard convection, Phys. Rev E 85, 016313 (2012).

[51] A. Sauret, D. Cébron, C. Morize, and M. Lebars, Experimental and numerical study of mean zonal flows generated by librations of a rotating spherical cavity, J. Fluid Mech. 662, 260 (2010).

[52] G. Bordes, A. Venaille, S. Joubaud, P. Odier, and T. Dauxois, Experimental observation of a strong mean flow induced by internal gravity waves, Phys. Fluids 24, 086602 (2012).

[53] B. R. Sutherland, Internal wave instability: Wave-wave versus wave-induced mean flow interactions, Phys. Fluids 18, 074107 (2006).

[54] M. J. Mercier, N. B. Garnier, and T. Dauxois, Reflection and diffraction of internal waves analyzed with the Hilbert transform, Phys. Fluids 20, 086601 (2008).

[55] C. R. Koudella and C. Staquet, Instability mechanisms of a two-dimensional progressive internal gravity wave, J. Fluid Mech. 548, 165 (2006). 